\title{
Space Telescope and Optical Reverberation Mapping Project. V. Optical Spectroscopic Campaign and Emission-line Analysis for NGC 5548
}

L. Pei ${ }^{1,2}$, M. M. Fausnaugh ${ }^{3}$, A. J. Barth ${ }^{1}$, B. M. Peterson ${ }^{3,4,5}$, M. C. Bentz ${ }^{6}$, G. De Rosa ${ }^{5}$, K. D. Denney ${ }^{3,4,88}$, M. R. Goad ${ }^{7}$, C. S. Kochanek ${ }^{3,4}$, K. T. Korista ${ }^{8}$, G. A. Kriss ${ }^{5,9}$, R. W. Pogge ${ }^{3,4}$, V. N. Bennert ${ }^{10}$, M. Brotherton ${ }^{11}$, K. I. Clubb ${ }^{12}$, E. Dalla Bontà ${ }^{13,14}$, A. V. Filippenko ${ }^{12}$, J. E. Greene ${ }^{15}$, C. J. Grier ${ }^{3,16,17}$, M. Vestergaard ${ }^{18,19}$, W. Zheng ${ }^{12}$, Scott M. Adams ${ }^{3,20}$, Thomas G. Beatty ${ }^{3,16,21}$, A. Bigley ${ }^{12}$, Jacob E. Brown ${ }^{22}$, Jonathan S. Brown ${ }^{3}$, G. Canalizo ${ }^{23}$, J. M. Comerford ${ }^{24}$, Carl T. Coker ${ }^{3}$, E. M. Corsini ${ }^{13,14}$, S. Croft ${ }^{12}$, K. V. Croxall ${ }^{3,4}$, A. J. Deason ${ }^{25}$, Michael Eracleous ${ }^{16,17,26,27}$, O. D. Fox ${ }^{12}$, E. L. Gates ${ }^{28}$, C. B. Henderson ${ }^{3,29,89}$, E. Holmbeck ${ }^{30}$, T. W.-S. Holoien ${ }^{3,4}$, J. J. Jensen ${ }^{18}$, C. A. Johnson ${ }^{31}$, P. L. Kelly ${ }^{32,33,34}$, S. Kim ${ }^{3,4}$, A. King ${ }^{35}$, M. W. Lau ${ }^{25}$, Miao Li ${ }^{36}$, Cassandra Lochhaass, Zhiyuan Ma ${ }^{22}$, E. R. Manne-Nicholas ${ }^{6}$, J. C. Mauerhan ${ }^{12}$, M. A. Malkan ${ }^{30}$, R. McGurk ${ }^{25,37}$, L. Morelli ${ }^{13,14}$, Ana Mosquera 3,38 , Dale Mudd ${ }^{3}$, F. Muller Sanchez ${ }^{24}$, M. L. Nguyen ${ }^{11}$, P. Ochner ${ }^{13,14}$, B. Ou-Yang ${ }^{6}$, A. Pancoast ${ }^{39,40,90}$, Matthew T. Penny ${ }^{3,91}$, A. Pizzella ${ }^{13,14}$, Radosław Poleski ${ }^{3}$, Jessie Runnoe ${ }^{16,17,41}$ B. Scott ${ }^{23}$, Jaderson S. Schimoia, 3,42 , B. J. Shappee ${ }^{43,92}$, I. Shivvers ${ }^{12}$, Gregory V. Simonian ${ }^{3}$, A. Siviero ${ }^{13}$, Garrett Somers ${ }^{3,44}$,' Daniel J. Stevens ${ }^{3}$, M. A. Strauss ${ }^{15}$, Jamie Tayar ${ }^{3}$, N. Tejos ${ }^{45,46}$, T. Treu ${ }^{30,39,93}$, J. Van Saders ${ }^{43}$, L. Vican ${ }^{30}$, S. Villanueva, Jr. ${ }^{3}$,

H. Yuk ${ }^{12}$, N. L. Zakamska ${ }^{9}$, W. Zhu ${ }^{3}$, M. D. Anderson ${ }^{6}$, P. Arévalo ${ }^{47}$, C. Bazhaw ${ }^{6}$, S. Bisogni ${ }^{3,48}$, G. A. Borman ${ }^{49}$, M. C. Bottorff $5^{50}$, W. N. Brandt ${ }^{16,17,51}$, A. A. Breeveld ${ }^{52}$, E. M. Cackett ${ }^{53}$, M. T. Carini ${ }^{54}$, D. M. Crenshaw ${ }^{6}$,

A. De Lorenzo-Cáceres ${ }^{55}$, M. Dietrich ${ }^{56,57}$, R. Edelson ${ }^{58}$, N. V. Efimova ${ }^{59}$, J. Ely ${ }^{5}$, P. A. Evans ${ }^{7}$, G. J. Ferland ${ }^{60}$, K. Flatland ${ }^{61}$, N. Gehrels ${ }^{62}$, S. Geier ${ }^{63,64,65}$, J. M. Gelbord ${ }^{66,67}$, D. Grupe ${ }^{68}$, A. Gupta ${ }^{3}$, P. B. Hall ${ }^{69}$, S. Hicks ${ }^{54}$, D. Horenstein ${ }^{6}$, Keith Horne ${ }^{55}$, T. Hutchison ${ }^{5}$, M. Im $^{70}$, M. D. Joner ${ }^{71}$, J. Jones ${ }^{6}$, J. Kaastra ${ }^{72,73,74}$, S. Kaspi ${ }^{75,76}$, B. C. Kelly ${ }^{39}$, J. A. Kennea ${ }^{16}$, M. Kim ${ }^{77}$,

S. C. $\mathrm{Kim}^{77}$, S. A. Klimanov ${ }^{60}$, J. C. Lee $^{77}$, D. C. Leonard ${ }^{61}$, P. Lira ${ }^{78}$, F. MacInnis ${ }^{50}$, S. Mathur ${ }^{3,4}$, I. M. McHardy ${ }^{79}$, C. Montouri ${ }^{80}$, R. Musso ${ }^{50}$, S. V. Nazarov ${ }^{49}$, H. Netzer ${ }^{75}$, R. P. Norris ${ }^{6}$, J. A. Nousek ${ }^{16}$, D. N. Okhmat ${ }^{49}$, I. Papadakis ${ }^{81,82}$, J. R. Parks ${ }^{6}$, J.-U. Pott ${ }^{37}$, S. E. Rafter ${ }^{76,83}$, H.-W. Rix ${ }^{37}$, D. A. Saylor ${ }^{6}$, K. Schnülle ${ }^{37}$, S. G. Sergeev ${ }^{49}$, M. Siegel ${ }^{84}$, A. Skielboe ${ }^{18}$, M. Spencer ${ }^{71}$, D. Starkey ${ }^{55}$, H.-I. Sung ${ }^{77}$, K. G. Teems ${ }^{6}$, C. S. Turner ${ }^{6}$, P. Uttley ${ }^{85}$, C. Villforth ${ }^{86}$, Y. Weiss ${ }^{76}$, J.-H. Woo ${ }^{70}$, H. Yan ${ }^{22}$, S. Young ${ }^{58}$, and $\mathrm{Y} . \mathrm{Zu}^{4,87}$

\footnotetext{
${ }^{1}$ Department of Physics and Astronomy, 4129 Frederick Reines Hall, University of California, Irvine, CA 92697, USA

${ }^{2}$ Department of Astronomy, University of Illinois at Urbana-Champaign, Urbana, IL 61801, USA

${ }^{3}$ Department of Astronomy, The Ohio State University, $140 \mathrm{~W}$ 18th Avenue, Columbus, OH 43210, USA

${ }^{4}$ Center for Cosmology and AstroParticle Physics, The Ohio State University, 191 West Woodruff Avenue, Columbus, OH 43210, USA

${ }^{5}$ Space Telescope Science Institute, 3700 San Martin Drive, Baltimore, MD 21218, USA

${ }^{6}$ Department of Physics and Astronomy, Georgia State University, 25 Park Place, Suite 605, Atlanta, GA 30303, USA

7 Department of Physics and Astronomy, University of Leicester, Leicester, LE1 7RH, UK

${ }^{8}$ Department of Physics, Western Michigan University, 1120 Everett Tower, Kalamazoo, MI 49008, USA

${ }_{9}$ Department of Physics and Astronomy, Johns Hopkins University, Baltimore, MD 21218, USA

${ }^{10}$ Physics Department, California Polytechnic State University, San Luis Obispo, CA 93407, USA

${ }^{11}$ Department of Physics and Astronomy, University of Wyoming, 1000 E. University Avenue, Laramie, WY 82071, USA

${ }^{13}$ Dipartimento di Fisica e Astronomia "G. Galilei," Università di Padova, Vicolo dell'Osservatorio 3, I-35122 Padova, Italy
${ }^{12}$ '

${ }^{14}$ INAF-Osservatorio Astronomico di Padova, Vicolo dell’Osservatorio 5 I-35122, Padova, Italy

${ }^{15}$ Department of Astrophysical Sciences, Princeton University, Princeton, NJ 08544, USA

${ }^{16}$ Department of Astronomy and Astrophysics, Eberly College of Science, The Pennsylvania State University, 525 Davey Laboratory, University Park, PA 16802, USA

${ }^{17}$ Institute for Gravitation and the Cosmos, The Pennsylvania State University, University Park, PA 16802, USA

${ }^{18}$ Dark Cosmology Centre, Niels Bohr Institute, University of Copenhagen, Juliane Maries Vej 30, DK-2100 Copenhagen Ø, Denmark

${ }^{19}$ Steward Observatory, University of Arizona, 933 North Cherry Avenue, Tucson, AZ 85721, USA

${ }^{20}$ Cahill Center for Astrophysics, California Institute of Technology, Pasadena, CA 91125, USA

${ }^{21}$ Center for Exoplanets and Habitable Worlds, The Pennsylvania State University, University Park, PA 16802, USA

${ }^{22}$ Department of Physics and Astronomy, University of Missouri, Columbia, MO 65211, USA ${ }^{23}$ Department of Astronomy, University of California, Riverside, CA 92521, USA

${ }^{24}$ Department of Astrophysical and Planetary Sciences, University of Colorado, Boulder, CO 80309, USA

${ }^{25}$ Department of Astronomy and Astrophysics, University of California Santa Cruz, 1156 High Street, Santa Cruz, CA 95064, USA

${ }^{26}$ Center for Relativistic Astrophysics, Georgia Institute of Technology, Atlanta, GA 30332, USA

${ }^{27}$ Department of Astronomy, University of Washington, Box 351580, Seattle, WA 98195, USA ${ }^{28}$ Lick Observatory, P.O. Box 85, Mt. Hamilton, CA 95140, USA

${ }^{29}$ Jet Propulsion Laboratory, California Institute of Technology, 4800 Oak Grove Drive, Pasadena, CA 91109, USA

${ }^{30}$ Department of Physics and Astronomy, University of California, Los Angeles, CA 90095, USA

${ }^{31}$ Santa Cruz Institute for Particle Physics and Department of Physics, University of California, Santa Cruz, CA 95064, USA

${ }^{32}$ Department of Physics, Stanford University, 382 Via Pueblo Mall, Stanford, CA 94305, USA

${ }^{33}$ Kavli Institute for Particle Astrophysics and Cosmology, Stanford University, Stanford, CA 94305, USA

${ }^{4}$ SLAC National Accelerator Laboratory, 2575 Sand Hill Road, Menlo Park, CA 94025, USA ${ }^{35}$ School of Physics, University of Melbourne, Parkville, VIC 3010, Australia

${ }^{36}$ Department of Astronomy, Columbia University, 550 W. 120th Street, New York, NY 10027, USA

${ }^{37}$ Max Planck Institut für Astronomie, Königstuhl 17, D-69117 Heidelberg, Germany

${ }^{38}$ Physics Department, United States Naval Academy, Annapolis, MD 21403, USA

${ }^{39}$ Department of Physics, University of California, Santa Barbara, CA 93106, USA

${ }^{40}$ Harvard-Smithsonian Center for Astrophysics, 60 Garden Street, Cambridge, MA 02138, USA

${ }^{41}$ Department of Astronomy, University of Michigan, 500 Church Street, Ann Arbor, MI 48109, USA
} 


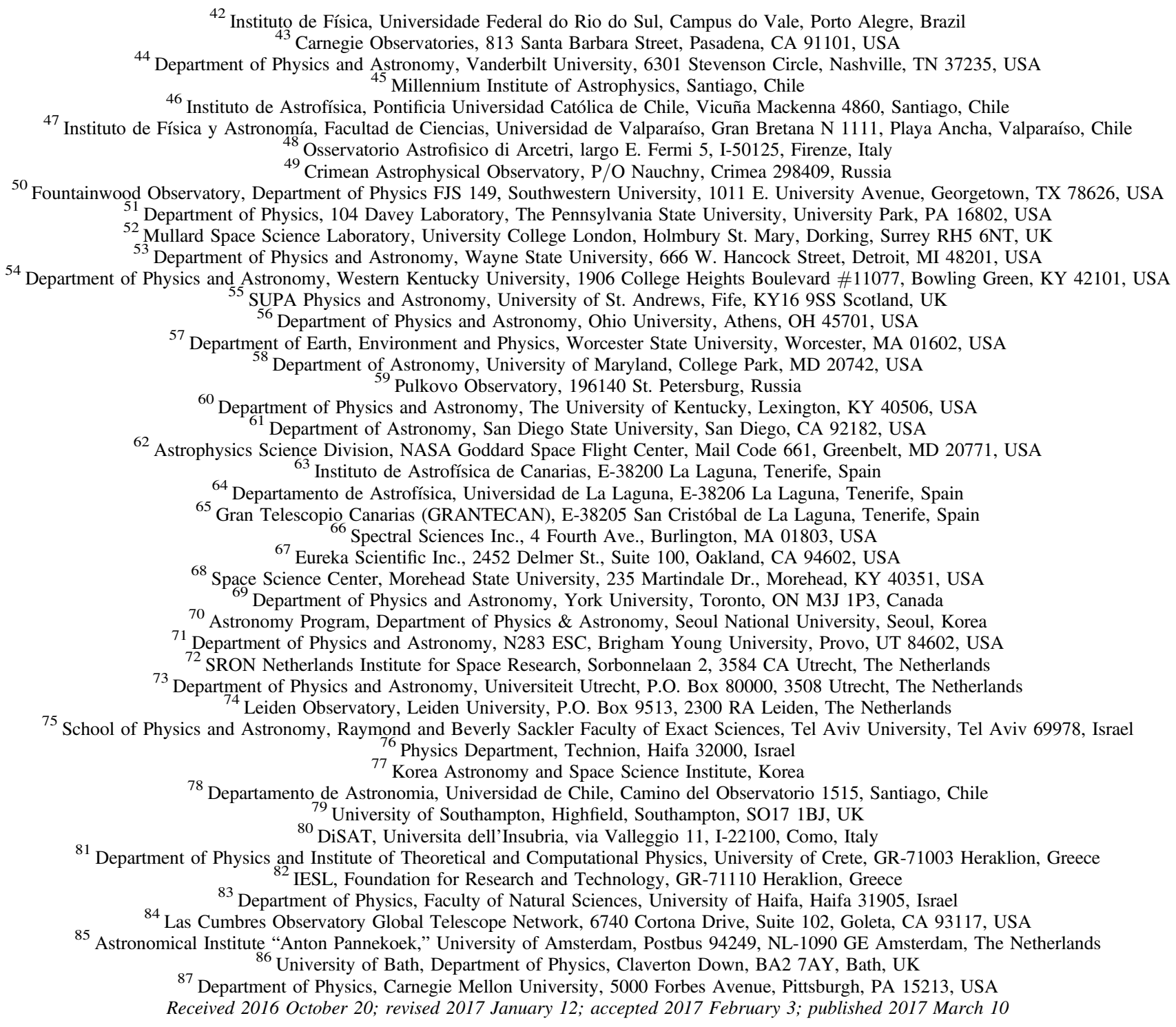

\begin{abstract}
We present the results of an optical spectroscopic monitoring program targeting NGC 5548 as part of a larger multiwavelength reverberation mapping campaign. The campaign spanned 6 months and achieved an almost daily cadence with observations from five ground-based telescopes. The $\mathrm{H} \beta$ and $\mathrm{He}$ II $\lambda 4686$ broad emission-line light curves lag that of the $5100 \AA$ optical continuum by $4.17_{-0.36}^{+0.36}$ days and $0.79_{-0.34}^{+0.35}$ days, respectively. The $\mathrm{H} \beta$ lag relative to the $1158 \AA$ Altraviolet continuum light curve measured by the Hubble Space Telescope is $\sim 50 \%$ longer than that measured against the optical continuum, and the lag difference is consistent with the observed lag between the optical and ultraviolet continua. This suggests that the characteristic radius of the broad-line region is $\sim 50 \%$ larger than the value inferred from optical data alone. We also measured velocity-resolved emission-line lags for $\mathrm{H} \beta$ and found a complex velocity-lag structure with shorter lags in the line wings, indicative of a broadline region dominated by Keplerian motion. The responses of both the $\mathrm{H} \beta$ and $\mathrm{He}$ II emission lines to the driving continuum changed significantly halfway through the campaign, a phenomenon also observed for C IV, Ly $\alpha$, He II (+O III]), and $\mathrm{Si} \mathrm{IV} \mathrm{(+O} \mathrm{IV])} \mathrm{during} \mathrm{the} \mathrm{same} \mathrm{monitoring} \mathrm{period.} \mathrm{Finally,} \mathrm{given} \mathrm{the} \mathrm{optical} \mathrm{luminosity} \mathrm{of} \mathrm{NGC} 5548$ during our campaign, the measured $\mathrm{H} \beta$ lag is a factor of five shorter than the expected value implied by the $R_{\mathrm{BLR}}{ }^{-}$ $L_{\mathrm{AGN}}$ relation based on the past behavior of NGC 5548 .
\end{abstract}

\footnotetext{
${ }^{88}$ NSF Postdoctoral Research Fellow.

89 NASA Postdoctoral Program Fellow.

${ }^{90}$ Einstein Fellow.

91 Sagan Fellow.

92 Carnegie-Princeton Fellow, Hubble Fellow.

93 Packard Fellow.
} 
Key words: galaxies: active - galaxies: individual (NGC 5548) - galaxies: nuclei - galaxies: Seyfert

Supporting material: machine-readable table

\section{Introduction}

Broad emission lines are among the most striking features of quasars and active galactic nuclei (AGNs). These Dopplerbroadened lines are emitted by gas occupying the broad-line region (BLR), which is located within several light-days to light-months of the central supermassive black hole $\mathrm{SMBH}$; e.g., Antonucci \& Cohen 1983; Clavel et al. 1991; Peterson et al. 1998, 2004; Bentz et al. 2009b; Grier et al. 2013). The geometry and kinematics of the BLR play a significant role in AGN research because these properties can be used to infer the mass of the central black hole (BH; e.g., Gaskell \& Sparke 1986; Clavel et al. 1991; Kaspi et al. 2000; Denney et al. 2006, 2010; Pancoast et al. 2014). Additionally, it is possible that infalling BLR gas may fuel SMBH accretion (e.g., Peterson 2006; Gaskell \& Goosmann 2016) and outflowing gas may be part of disk winds that carry away angular momentum from the disk and provide energy and momentum feedback to the host galaxy (e.g., Emmering et al. 1992; Murray \& Chiang 1997; Kollatschny 2003; Leighly \& Moore 2004). Understanding the dynamical state and physical conditions of gas in the BLR is of key importance in completing our understanding of the AGN phenomenon.

Owing to its small angular size, the BLR is currently impossible to resolve spatially even for the closest AGNs. An alternative method to study this region is to resolve it in the time domain using reverberation mapping (RM), a technique that leverages the variable nature of quasars and Seyferts (Blandford \& McKee 1982; Peterson 1993, 2014). AGNs exhibit stochastic flux variations, possibly because of inhomogeneous accretion and thermal fluctuations in the accretion disk (Czerny et al. 1999, 2003Collier \& Peterson 2001; Kelly et al. 2009; Kozłowski et al. 2010; MacLeod et al. 2010). Photons from the central engine ionize the BLR gas, which then echoes continuum flux variations with a light-travel time lag, $\tau$. The emission-line flux $L\left(v_{r}, t\right)$ at time $t$ and line-of-sight velocity $v_{r}$ is related to the ionizing continuum by

$$
L\left(v_{r}, t\right)=\int_{0}^{\infty} \Psi\left(v_{r}, \tau\right) C(t-\tau) d \tau,
$$

where $C(t-\tau)$ is the continuum emission at an earlier time $t-\tau$, and $\Psi(v, \tau)$ is the transfer function that maps the continuum light curve to the time-variable line profile (Blandford \& McKee 1982).

The transfer function-also known as the velocity-delay map - encodes important information about the BLR's geometry and kinematics. There has been tremendous effort by many groups to recover velocity-delay maps (Rosenblatt \& Malkan 1990; Horne et al. 1991; Krolik et al. 1991; Ulrich \& Horne 1996; Bentz et al. 2010a; Pancoast et al. 2011, 2014; Grier et al. 2013; Li et al. 2013; Skielboe et al. 2015) and velocity-resolved line lags (e.g., Kollatschny 2003; Bentz et al. 2009b; Denney et al. 2010; Barth et al. 2011; Du et al. 2016a). In order to obtain $\Psi(v, \tau), \mathrm{RM}$ campaigns must have a combination of high cadence, long duration, high photometric precision, and high signal-to-noise ratios $(\mathrm{S} / \mathrm{Ns})$, which is often not achievable by ground-based programs. More typically, RM campaigns are able to only measure the mean emission-line lag $\tau$, which represents the response-weighted mean light-travel time from the ionizing continuum to the BLR.

Assuming that the broad-line width is a result of the virialized motion of gas within the BH's potential well, the emission-line lag and gas velocity dispersion inferred from the line width $(\Delta V)$ can be used to infer the $\mathrm{BH}$ mass using

$$
M_{\mathrm{BH}}=f \frac{c \tau \Delta V^{2}}{G} .
$$

Here, $c \tau=R_{\mathrm{BLR}}$ is the characteristic radius of the BLR, and $f$ is a dimensionless calibration factor of order unity that accounts for the unknown BLR geometry and kinematics. Ground-based RM campaigns have produced BH mass measurements for $\sim 60$ local AGNs to date (see Bentz \& Katz 2015 , for references and a recent compilation). RM is also starting to be used for objects at cosmological distances (Kaspi et al. 2007; King et al. 2015; Shen et al. 2016), with the aims of studying the UV continuum and emission lines and calibrating $\mathrm{BH}$ masses at high redshifts.

The ionizing continuum is emitted at wavelengths $<912 \AA$ and is generally unobservable, due to the Lyman limit of the host galaxy. Given this limitation, the far-UV continuum at $\lambda \approx 1100-1500 \AA$ should be used to derive emission-line lags because it is close in wavelength to the ionizing continuum and should therefore serve as an accurate proxy. However, wavelengths shorter than $\sim 3200 \AA$ are inaccessible from the ground, so the rest-frame optical continuum is often used as a proxy for the ionizing source in low-redshift AGNs. Although the far-UV and optical continua have been shown to vary almost simultaneously in some cases (e.g., Clavel et al. 1991; Reichert et al. 1994; Korista et al. 1995; Wanders et al. 1997), more recent high-cadence studies have found that the optical continuum can lag the UV continuum by up to a few days (Collier et al. 1998; Sergeev et al. 2005; McHardy et al. 2014; Shappee et al. 2014; Edelson et al. 2015; Fausnaugh et al. 2016). This can significantly affect the measured broadline lag if the BLR has a characteristic radius on the order of light-days. The variable optical continuum has also been shown to have smoother features and smaller amplitudes than its UV counterpart (e.g., Peterson et al. 1991; Dietrich et al. 1993, 1998; Stirpe et al. 1994; Santos-Lleó et al. 1997; Shappee et al. 2014; Fausnaugh et al. 2016). These differences between the UV and optical continua suggest that the optical continuum is not fully interchangeable with the ionizing source for determining reverberation lags.

Furthermore, a long-standing assumption in RM is that the source of the ionizing photons in a typical Seyfert galaxy is physically much smaller than the BLR (about a factor of 100; e.g., Peterson 1993; Peterson \& Horne 2004). This assumption implies that the disk size can be neglected when determining $R_{\mathrm{BLR}}$ from RM data. However, Fausnaugh et al. (2016) have shown that the optically emitting portion of the accretion disk has a lag similar to that of the inner portion of the BLR. If we assume a model in which the measured lags are purely dependent on the radial distance from the ionizing source, then the emission-line lags measured using the optical continuum may significantly underestimate the BLR characteristic radius. 
Table 1

Instrument Characteristics and Data Reduction Parameters for All Telescopes

\begin{tabular}{|c|c|c|c|c|c|c|c|c|}
\hline Telescope & Instrument & $\begin{array}{c}\text { Number of } \\
\text { Epochs }\end{array}$ & $\begin{array}{l}\text { Median } \\
\text { Seeing } \\
(\operatorname{arcsec})\end{array}$ & $\begin{array}{l}\text { Wavelength } \\
\text { Dispersion } \\
\left(\AA \text { pixel }^{-1}\right)\end{array}$ & $\begin{array}{c}\text { Wavelength } \\
\text { Coverage } \\
\text { (̊) }\end{array}$ & $\begin{array}{c}\text { Pixel } \\
\text { Scale } \\
\left(\operatorname{arcsec~pixel~}^{-1}\right)\end{array}$ & $\begin{array}{c}\text { Median } \\
\mathrm{S} / \mathrm{N}\end{array}$ & $\begin{array}{c}{[\mathrm{O} \mathrm{III}]} \\
F_{\mathrm{var}} \\
(\%)\end{array}$ \\
\hline MDM & Boller \& Chivens CCD Spectrograph & 143 & 1.7 & 1.25 & $4225-5775$ & 0.75 & 118 & 0.62 \\
\hline Lick & Kast Double Spectrograph & 35 & 1.5 & 1.02 & $3460-5500$ & 0.43 & 194 & 0.32 \\
\hline Asiago & Boller \& Chivens CCD Spectrograph & 21 & 4.0 & 1.00 & $3250-7920$ & 1.00 & 160 & 0.27 \\
\hline APO & Dual Imaging Spectrograph & 13 & 1.4 & 1.00 & $4180-5400$ & 0.41 & 160 & 0.28 \\
\hline WIRO & WIRO Long Slit Spectrograph & 6 & 2.1 & 0.74 & $5599-4399$ & 0.52 & 217 & 0.47 \\
\hline
\end{tabular}

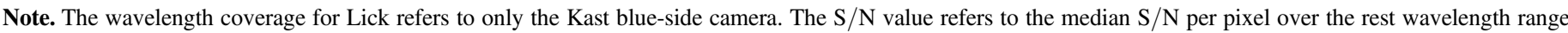

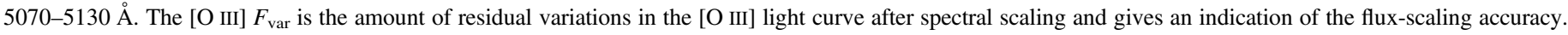

Since most RM campaigns use only optical data, it is imperative that we understand the systematic effects of using the optical rather than the UV continuum in RM studies and the relevant implications for $\mathrm{BH}$ mass estimates.

To this end, we present the results of a 6-month groundbased RM program monitoring the galaxy NGC 5548 (redshift $z=0.0172$ ). This paper is the fifth in a series describing results from the AGN Space Telescope and Optical Reverberation Mapping (AGN STORM) campaign, the most intensive multiwavelength AGN monitoring program to date. The campaign is centered around 171 epochs of daily cadence observations using the Cosmic Origins Spectrograph on the Hubble Space Telescope (HST). Concurrent with the HST program were 4 months of Swift observations and 6 months of ground-based photometric and spectroscopic observations. First results of the HST, Swift, and ground-based photometry programs were presented by De Rosa et al. (2015), Edelson et al. (2015), and Fausnaugh et al. (2016) (Papers I-III, respectively). Goad et al. (2016) (Paper IV) explore the anomalous behavior of the UV continuum and broad emission-line light curves observed during a portion of this campaign. This paper focuses on the ground-based spectroscopic data and emission-line analysis.

NGC 5548 is one of the best-studied Seyfert galaxies and has been the subject of many past RM programs. Most notably, it was the target of a 13 yr campaign carried out by the AGN Watch consortium (Peterson et al. 2002, and references therein), which was initially designed to support UV monitoring of NGC 5548 carried out by the International Ultraviolet Explorer (IUE; Clavel et al. 1991). Individual years of this campaign achieved median sampling cadences of 1-3 days for spectroscopic observations. Subsequently, NGC 5548 was monitored in programs described by Bentz et al. (2007), Denney et al. (2009), Bentz et al. (2009b), and G. De Rosa et al. (2017, in preparation) with campaign durations of 40, 135, 64, and 120 days (respectively), and each with a median sampling cadence of $\sim 1$ day. A more recent RM program described by Lu et al. (2016) monitored this AGN for 180 days with a median spectroscopic sampling of $\sim 3$ days. The 2014 AGN STORM campaign's combination of daily cadence, 6month duration, and multiwavelength coverage makes it the most intensive RM campaign ever conducted.

There are two primary goals of the present work. The first is to compare the $\mathrm{H} \beta$ emission-line lag measured against simultaneously observed far-UV and optical continua in order to understand the effects of substituting the optical continuum for the ionizing continuum in reverberation measurements. The second goal is to examine in detail the responses of the optical emission lines to continuum variations and compare them to those of the UV lines, which will provide a more complete picture of the structure and kinematics of the BLR than previous studies that used only optical data.

We describe the spectroscopic observations and reductions in Section 2. Section 3 details our procedures for flux and light-curve measurements. In Section 4, we present our analysis of emissionline lags, line responses, line profiles, and $\mathrm{BH}$ mass measurements. We discuss the implications of our results and compare our measurements with those from previous campaigns in Section 5. Section 6 summarizes our findings. We quote wavelengths in the rest frame of NGC 5548 unless otherwise stated.

\section{Observations and Data Reduction}

Spectroscopic data were obtained from five telescopes: the McGraw-Hill $1.3 \mathrm{~m}$ telescope at the MDM Observatory, the Shane $3 \mathrm{~m}$ telescope at the Lick Observatory, the $1.22 \mathrm{~m}$ Galileo telescope at the Asiago Astrophysical Observatory, the $3.5 \mathrm{~m}$ telescope at Apache Point Observatory (APO), and the $2.3 \mathrm{~m}$ telescope at the Wyoming Infrared Observatory (WIRO). Observations at MDM were carried out with a slit width of $5^{\prime \prime}$ oriented in the north-south direction, and spectra at the other telescopes were taken with a $5^{\prime \prime}$-wide slit oriented at the parallactic angle (Filippenko 1982). The optical spectroscopic monitoring began on 2014 January 4 (UT dates are used throughout this paper) and continued through 2014 July 6 with approximately daily cadence.

Table 1 lists the properties of the telescopes and instruments used to obtain spectroscopic data, and Figure 1 shows the mean spectrum constructed using data from Asiago, which obtained the only spectra that cover the full optical wavelength range. MDM contributed the largest number of spectra with 143 epochs. The 35 epochs of Lick spectra were obtained by several groups of observers who used slightly different setups and calibrations. The Kast spectrograph (Miller \& Stone 1993) at Lick Observatory has red-side and blue-side cameras, but since the red-side setup was very different for each group, we present only the blue-side data here. Asiago, APO, and WIRO contributed 21, 13, and 6 epochs of spectra, respectively. Our analysis focuses primarily on the MDM data set for homogeneity.

Data reduction procedures included bias subtraction, flatfielding, and cosmic-ray removal using the L.A. Cosmic routine (van Dokkum 2001). The one-dimensional spectra were extracted from a $15^{\prime \prime}$-wide region centered on the AGN and with consistent background sky apertures for all observations. We used optimally weighted extractions for the stellar spectra 


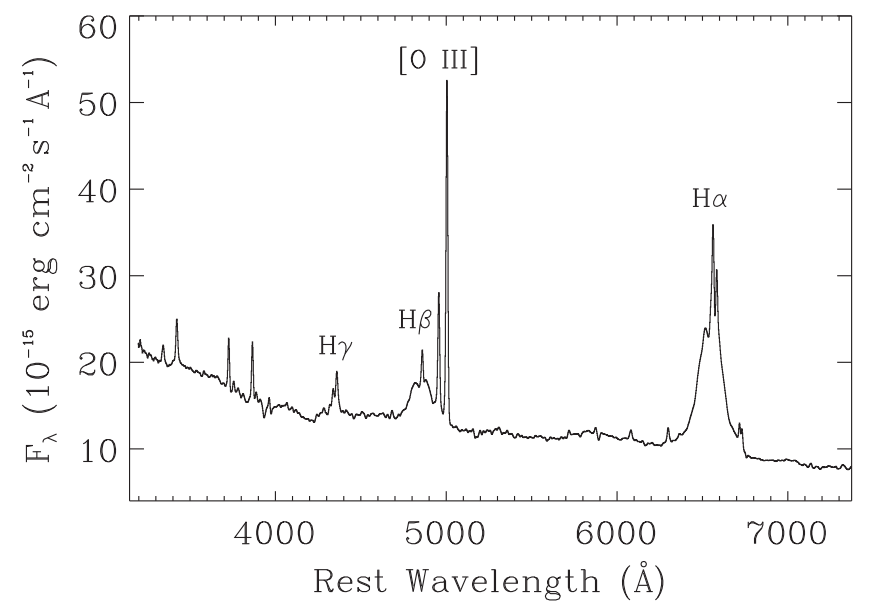

Figure 1. Mean spectrum of NGC 5548 from the Asiago data set, which includes 21 epochs of spectra with spectral resolution of $1.0 \AA \mathrm{Aixel}^{-1}$ and has a median $\mathrm{S} / \mathrm{N}$ of 160 . Labeled are the He II $\lambda 4686, \mathrm{H} \beta \lambda 4861$, and [O III] $\lambda \lambda 4959,5007$ emission lines.

(Horne 1986) but unweighted extractions for the AGN spectra. This is because the optimal extraction method requires the spatial profile of the target to be a smooth function of wavelength and tends to truncate the peaks of strong emission lines such as [O III] that have different spatial extents from the surrounding continuum.

The data were wavelength-calibrated using night-sky lines and flux-calibrated using standard stars. Our most frequently used flux standard stars were Feige 34, BD 332642, and HZ 44. For nights when multiple exposures were taken, we aligned the flux-calibrated one-dimensional spectra by applying small wavelength shifts to each spectrum before combining them. We do not expect significant differential atmospheric refraction (Filippenko 1982) because of the large slit width used for our observations.

For the MDM data, the first 133 epochs were flux-calibrated using Feige 34, while the last 10 epochs, taken from 2014 June 20 to 2014 June 30, were flux-calibrated with BD 332642. This caused spurious changes in the shape of some emission-line features, so we use only the first 133 MDM epochs for our present analysis.

\subsection{Spectral Flux Calibrations}

To place the instrumental fluxes on an absolute flux scale, we measured the narrow [O III] $\lambda 5007$ line flux from spectra taken under photometric conditions and scaled all other nightly spectra to have the same [O III] flux. There were 21 epochs identified as having been observed under photometric conditions by the MDM observers. We determined the flux of the [O III] line ( $\lambda_{\text {observed }}=5093 \AA$ ) by first subtracting a linear fit to continuum windows on either side of the line and then integrating over a fixed wavelength range. We used the rest-frame wavelength ranges $4976.5-4948.0 \AA$ and $5027.7-5031.6 \AA$ to fit the continuum and integrated over the range 4980.5-5026.7 $\AA$ for the line flux. The $2 \sigma$ outliers from this set of [O III] flux measurements were discarded, the mean was recomputed, and this process was repeated until there were no more $2 \sigma$ outliers, which resulted in a total of 16 final photometric spectra. The mean spectrum of these 16 epochs has an [O III] $\lambda 5007$ line flux of $(5.01 \pm 0.11) \times 10^{-13} \mathrm{erg} \mathrm{s}^{-1} \mathrm{~cm}^{-2}$, which represents our best estimate of the true [O III] flux for NGC 5548 during this campaign and is not expected to vary over a 6-month period. For comparison, Peterson et al. (2013) found the [O III] flux in NGC 5548 to be $(4.77 \pm 0.14) \times 10^{-13} \mathrm{erg} \mathrm{s}^{-1} \mathrm{~cm}^{-2}$ in their 2012 monitoring campaign, and the difference is within the range of total [O III] variability observed for NGC 5548 over the course of 21 yr (see Peterson et al. 2013).

In addition to the intrinsic variability of the AGN, many other factors contribute to nightly variations in the spectra. These include changes in transparency due to clouds, changes in seeing conditions, inconsistent instrument focus, and miscentering of the AGN in the slit during observations. We used the flux-scaling method described by van Groningen \& Wanders (1992) to align the nightly spectra and place them on a consistent flux scale. For each spectrum in the data set, the algorithm looks for a combination of wavelength shift, multiplicative scale factor, and Gaussian kernel convolution that minimizes the residual between each individual spectrum and a reference spectrum over a region containing the narrow [O III] line.

We constructed a separate reference spectrum for each telescope by averaging the highest-S/N spectra in each data set and then broadened the reference spectrum so that the [O III] line width matches the broadest [O III] line width in the data set. This extra broadening of the reference spectrum helps to reduce the [O III] residuals from spectral scaling (Fausnaugh 2016). We then scaled each spectrum to have the same [O III] flux as the photometrically calibrated mean MDM spectrum. This brings all spectra to a common flux scale after spectral scaling.

To assess the accuracy of spectral scaling, we estimated the intrinsic fractional variability of the residual [O III] $\lambda 5007$ light curve after correcting for random measurement errors,

$$
F_{\mathrm{var}}=\frac{\sqrt{\sigma^{2}-\left\langle\delta^{2}\right\rangle}}{\langle f\rangle},
$$

where $\sigma^{2}$ is the [O III] flux variance, $\left\langle\delta^{2}\right\rangle$ is the mean-square value of the measurement uncertainties determined from the nightly error spectra produced by the data reduction pipeline, and $\langle f\rangle$ is the unweighted mean flux. The $F_{\text {var }}$ for the [O III] $\lambda 5007$ light curve gives a good estimate of the residual fluxscaling errors (Barth \& Bentz 2016), and the value for each telescope is listed in the last column of Table 1 . We found $F_{\text {var }}$ to be between $0.27 \%$ and $0.62 \%$ for all telescopes, which means that there is an additional scatter of less than $1 \%$ in the [O III] light curve above the measurement errors. These $F_{\text {var }}$ values are consistent with or better than the best values typically obtained in ground-based campaigns. For example, Barth et al. (2015) found $F_{\text {var }}$ values ranging from $0.5 \%$ to $3.3 \%$ for individual AGNs in the 2011 Lick AGN Monitoring Project.

Figure 2 shows (in black) the mean and rms residual spectra for the MDM data set. The rms spectrum indicates the degree of variability at each wavelength over the course of the campaign. Both the broad $\mathrm{H} \beta$ and $\mathrm{He}$ II $\lambda 4686$ emission lines exhibit strong variations, and the $\mathrm{H} \beta \mathrm{rms}$ profile appears to have multiple peaks. Traditionally, the rms spectrum is constructed such that the value at each wavelength is taken to be the standard deviation of fluxes from all epochs, but this does not take into account Poisson or detector noise, which may bias the rms profile by a small amount (Barth et al. 2015). Park et al. (2012b) suggest using the $\mathrm{S} / \mathrm{N}$ for each spectrum as the weight for that spectrum in calculating the rms, or using a 


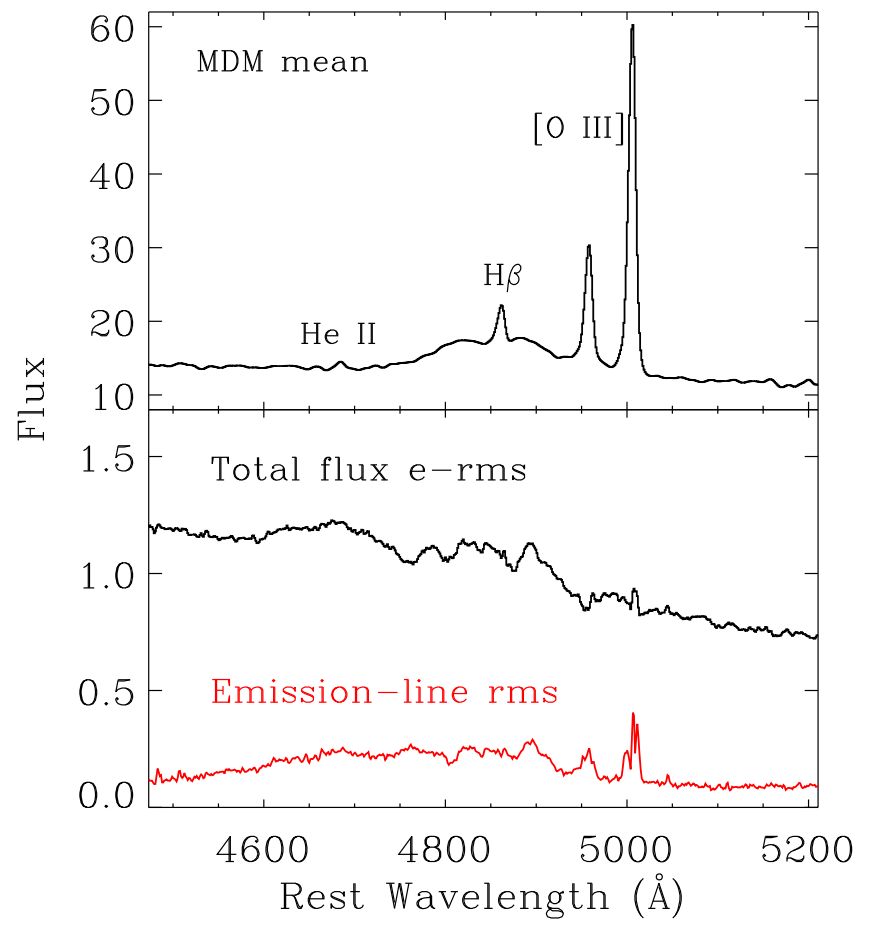

Figure 2. Mean and excess rms (Equation (4)) spectra from the MDM data set are shown in black, and the rms spectrum with the AGN and stellar continuum removed is shown in red (see Section 3.1).

maximum-likelihood method to obtain the rms. We adopt a simpler approach that uses the excess variance as a way to exclude variations that are not intrinsic to the AGN. This "excess rms" value at each wavelength is defined as

$$
\mathrm{e}-\mathrm{rms}_{\lambda}=\sqrt{\frac{1}{N-1} \sum_{i=1}^{N}\left[\left(F_{\lambda, i}-\left\langle F_{\lambda}\right\rangle\right)^{2}-\delta_{\lambda, i}^{2}\right]}
$$

where $N$ is the total number of spectra in the data set, $\left\langle F_{\lambda}\right\rangle$ is the mean flux at each wavelength, and $F_{\lambda, i}$ and $\delta_{\lambda, i}$ are the wavelength-specific fluxes and associated measurement uncertainties from individual epochs, respectively. This method estimates the degree of variability above what is expected given the measurement uncertainties and pixel-to-pixel noise.

\section{Spectroscopic Flux Measurements}

The $5100 \AA$ continuum flux density was determined by averaging the flux over the rest-frame wavelength range 5070-5130 $\AA$. The $\mathrm{H} \beta$ line fluxes were measured from the scaled spectra using the same method as for [O III] $\lambda 5007$, where we subtracted a linear fit to the surrounding continuum (wavelength windows 4483.0-4542.0 $\AA$ and 5033.5-5092.5 $\AA$ ) and integrated across the line profile (4748.4-4945.1 $\AA$ ). The uncertainty in each measurement is a combination of Poisson noise and residuals from spectral scaling. We computed the spectral scaling uncertainty by multiplying each flux measure-

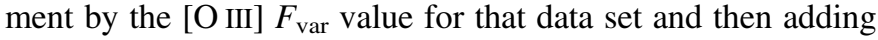
this value in quadrature to the Poisson noise to obtain the final flux uncertainty for each measurement. There is an additional source of spectral scaling uncertainty from slight differences in the overall spectral shape from night to night. This effect is likely small for $\mathrm{H} \beta$ because it is very close to the [O III] $\lambda 5007$ line that anchors the spectral scaling.

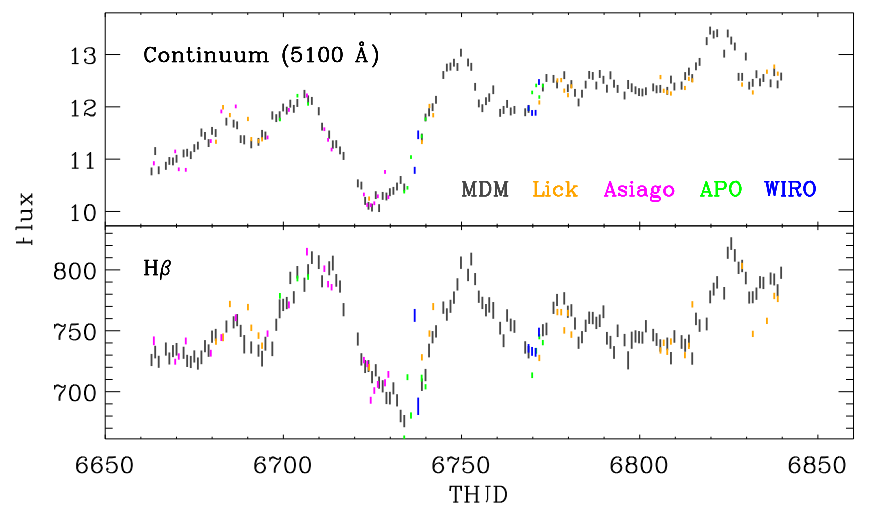

Figure 3. Continuum $\left(10^{-15} \mathrm{erg} \mathrm{s}^{-1} \mathrm{~cm}^{-2} \AA^{-1}\right)$ and $\mathrm{H} \beta\left(10^{-15} \mathrm{erg} \mathrm{s}^{-1} \mathrm{~cm}^{-2}\right)$ light curves (THJD $=$ HJD $-2,450,000$ ). The Lick, APO, Asiago, and WIRO light curves were scaled and shifted to match the MDM light curve, which has the longest temporal coverage and highest sampling cadence. The plotted uncertainties include Poisson noise and the normalized excess variance of the [O III] light curve (Section 2.1).

Spectrophotometric calibrations of the reference spectra, as described in the previous section, converted all instrumental fluxes to absolute fluxes, which means that measurements from all telescopes should now be on the same flux scale. However, light curves from different observing sites may be offset from each other owing to aperture effects (Peterson et al. 1995, 1999). While our observations were standardized to have the same $5^{\prime \prime} \times 15^{\prime \prime}$ aperture size, significant differences in image quality between observing sites could still cause flux offsets.

To intercalibrate the $\mathrm{H} \beta$ light curves, we used data points from each non-MDM telescope $\left(F_{\mathrm{H} \beta, \mathrm{t}}\right)$ that are nearly contemporaneous with MDM observations $\left(F_{\mathrm{H} \beta, \mathrm{MDM}}\right)$ and performed a least-squares fit to the equation

$$
F_{\mathrm{H} \beta, \mathrm{MDM}}=\phi F_{\mathrm{H} \beta, \mathrm{t}}
$$

to find the scale factor $\phi$ that puts each line light curve on the same flux scale as the MDM data. For the continuum intercalibration, we also include an additive shift $G$ to account for the differences in the host-galaxy flux admitted by different apertures:

$$
F_{5100, \mathrm{MDM}}=\phi F_{5100, \mathrm{t}}+G
$$

The scale factors for the Lick, Asiago, APO, and WIRO light curves are $\phi=[0.961,0.963,1.037,0.918]$, and the shift constants are $G=[-0.155,-0.640,-0.041,0.024]$ in units of $10^{-15} \mathrm{erg} \mathrm{s}^{-1} \mathrm{~cm}^{-2} \AA^{-1}$. The combined continuum and $\mathrm{H} \beta$ light curves are shown in Figure 3 (THJD $=$ HJD $-2,450,000$ ), and the $5100 \AA$ continuum and $\mathrm{H} \beta$ fluxes are listed in Table 2 .

We attempted to measure the He II $\lambda 4686$ flux from the nightly spectra. However, this line is very weak and also heavily blended with the broad $\mathrm{H} \beta$, as shown in Figure 2. Thus, we were unable to obtain an He II light curve using the linear interpolation method to remove the continuum.

\subsection{Spectral Decomposition}

To more accurately remove the continuum underlying the emission lines and to deblend the broad emission features from each other, we employed the spectral decomposition algorithm described by Barth et al. (2015). The components fitted in this procedure include narrow [O III], broad and narrow $\mathrm{H} \beta$, broad 
Table 2

Flux Measurements for Continuum and Emission Lines

\begin{tabular}{|c|c|c|c|c|c|}
\hline HJD $-2,450,000$ & Telescope & $F_{5100}$ & $F_{\mathrm{H} \beta}$ & $F_{\mathrm{H} \beta, \mathrm{SD}}$ & $F_{\mathrm{He} \text { II,SD }}$ \\
\hline 6663.00 & MDM & $10.766 \pm 0.075$ & $726.012 \pm 4.985$ & $710.187 \pm 4.897$ & $21.720 \pm 2.606$ \\
\hline 6663.65 & Asiago & $10.921 \pm 0.040$ & $741.771 \pm 3.586$ & $\ldots$ & $\ldots$ \\
\hline 6664.03 & MDM & $11.154 \pm 0.075$ & $732.511 \pm 5.156$ & $715.057 \pm 5.061$ & $28.154 \pm 3.222$ \\
\hline 6665.02 & MDM & $10.788 \pm 0.075$ & $724.537 \pm 4.946$ & $709.473 \pm 4.860$ & $25.135 \pm 2.451$ \\
\hline 6667.02 & MDM & $10.872 \pm 0.076$ & $735.001 \pm 5.393$ & $711.347 \pm 5.288$ & $37.008 \pm 3.964$ \\
\hline 6668.00 & MDM & $10.966 \pm 0.075$ & $727.261 \pm 4.946$ & $708.252 \pm 4.859$ & $35.227 \pm 2.450$ \\
\hline 6669.01 & MDM & $10.956 \pm 0.075$ & $733.312 \pm 4.941$ & $714.465 \pm 4.855$ & $40.589 \pm 2.430$ \\
\hline 6669.65 & Asiago & $11.147 \pm 0.035$ & $724.604 \pm 2.449$ & $\ldots$ & $\ldots$ \\
\hline 6670.02 & MDM & $11.008 \pm 0.076$ & $734.810 \pm 5.179$ & $712.584 \pm 5.083$ & $42.710 \pm 3.308$ \\
\hline 6670.65 & Asiago & $10.806 \pm 0.035$ & $728.863 \pm 2.626$ & $\ldots$ & $\ldots$ \\
\hline
\end{tabular}

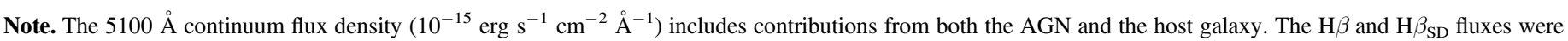

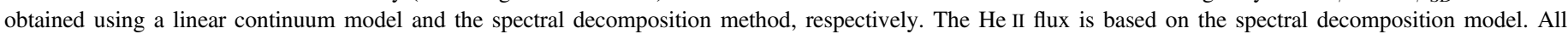
emission-line fluxes are in units of $10^{-15} \mathrm{erg} \mathrm{s}^{-1} \mathrm{~cm}^{-2}$ and include contributions from both broad- and narrow-line components.

(This table is available in its entirety in machine-readable form.)

and narrow He II, Fe II emission blends, the stellar continuum, and the AGN continuum. The host-galaxy starlight was modeled with an $11 \mathrm{Gyr}$, solar-metallicity, single-burst spectrum from Bruzual \& Charlot (2003). For the Fe II model component, we tested three different templates from Boroson \& Green (1992), Véron-Cetty et al. (2004), and Kovačević et al. (2010). The Fe II templates were broadened by convolution with a Gaussian kernel in velocity. The free fit parameters for Fe II include the velocity shift relative to broad $\mathrm{H} \beta$, the broadening kernel width, and the flux normalization of the broadened template spectrum. The Boroson \& Green (1992) and Véron-Cetty et al. (2004) templates are monolithic and require only one flux normalization parameter, whereas the Kovačević et al. (2010) template has five components that can vary independently in flux. The Kovačević et al. (2010) template achieves the best fit to the nightly spectra, presumably a result of the larger number of free fit parameters due to the multicomponent Kovačević et al. (2010) template.

We made several modifications to the spectral fitting procedures used by Barth et al. (2015). First, because of the complex line profiles, we used sixth-order Gauss-Hermite functions (van der Marel \& Franx 1993) to fit the broad and narrow $\mathrm{H} \beta$ and narrow [O III] lines instead of fourth-order functions. Second, there is significant degeneracy between the weak Fe II blend and the continuum flux in the nightly fits. Since the Fe II fit is poorly constrained and sometimes varied drastically from night to night, the continuum model flux also varied significantly as a result, which in turn introduced noise to the broad $\mathrm{H} \beta$ fit component. To address this issue, we constrained the Fe II flux to lie within $10 \%$ of the value from the fit to the mean spectrum (Barth et al. 2013). We also fixed the Fe II redshift to that of the mean spectrum and constrained the Fe II broadening kernel to be within $5 \%$ of its value from the mean spectrum fit. The He I $\lambda 4922$ and $\lambda 5016$ lines are very weak and are heavily blended with broad $\mathrm{H} \beta$, making it impossible to constrain their fit parameters. We therefore do not fit for these components in our model.

The broad He II $\lambda 4686$ component has very low amplitude compared to the other fit components, and it is blended with the blue wing of broad $\mathrm{H} \beta$. It is also highly variable, as demonstrated by the broad bump in the rms spectrum. This made it difficult to fit the He II broad-line profile accurately, and the width varied significantly from night to night when fitted as a free parameter. Since the He II $\lambda \lambda 1640$ and 4868 lines are expected to form under the same physical conditions and should thus have similar widths, we used fits to the $\lambda 1640$ line in concurrent HST spectra to constrain the $\lambda 4686$ line width.

The He II $\lambda 1640$ line was modeled with five Gaussian components (G. De Rosa et al. 2017, in preparation), and we took the three broadest components to represent the broad He II $\lambda 1640$ line profile. For each MDM spectrum, the He II $\lambda 4686$ broad-line FWHM was allowed to vary within $3 \AA$ of the He II $\lambda 1640$ FWHM measured from the closest HST epoch. The first 23 epochs from the MDM campaign do not have corresponding $H S T$ spectra, so for each of these "pre-HST" epochs, we found the three epochs from later in the campaign with the closest matching $5100 \AA$ continuum flux density. We then used the weighted mean of the broad He II $\lambda 1640$ widths from these three nights as the width constraint for the pre-HST epoch, where the weights were determined by how closely the $5100 \AA$ fluxes of the later epochs matched that of the pre-HST epoch. The He II $\lambda 1640$ line width was highly variable during the $H S T$ campaign, and the model FWHM widths used to constrain the spectral decomposition have a mean of $48 \AA$, with a minimum of $28 \AA$ and maximum of $59 \AA$.

We applied spectral decomposition to the data from all telescopes, but since the MDM data set is the largest and has the highest data quality and consistency, we use this data set for all subsequent analysis. Figure 4 shows the fit components for the mean MDM spectrum, where the black spectrum is the data and the red spectrum is the sum of all the model components. The model does not fit the detailed structure of the broad $\mathrm{H} \beta$ line well, especially in the line core. To prevent this from impacting our measured $\mathrm{H} \beta$ fluxes, we subtracted all the other well-modeled fit components except the broad and narrow $\mathrm{H} \beta$ components from the full spectrum and then obtained the $\mathrm{H} \beta$ line flux by integrating over the same wavelength range used to measure the flux without spectral decomposition. The He II $\lambda 4686$ flux was taken to be the total flux in the broad- and narrow-line models for each night. The narrow $\mathrm{H} \beta$ and He II $\lambda 4686$ line fluxes from fits to the mean spectrum are $48.4 \times 10^{-15} \mathrm{erg} \mathrm{s}^{-1} \mathrm{~cm}^{-2}$ and $8.5 \times 10^{-15} \mathrm{erg} \mathrm{s}^{-1} \mathrm{~cm}^{-2}$ (respectively), with uncertainties of $\sim 2 \%$ from the overall photometric scale of the data. The ratio of the narrow $\mathrm{H} \beta$ flux to the [O III] $\lambda 5007$ flux is $F_{\mathrm{H} \beta} / F_{[\mathrm{O} \text { III }]}=0.099 \pm 0.002$, which is in good agreement with the value of $F_{\mathrm{H} \beta} / F_{[\mathrm{O} \text { III] }}=$ $0.110 \pm 0.010$ found by Peterson et al. (2004). 


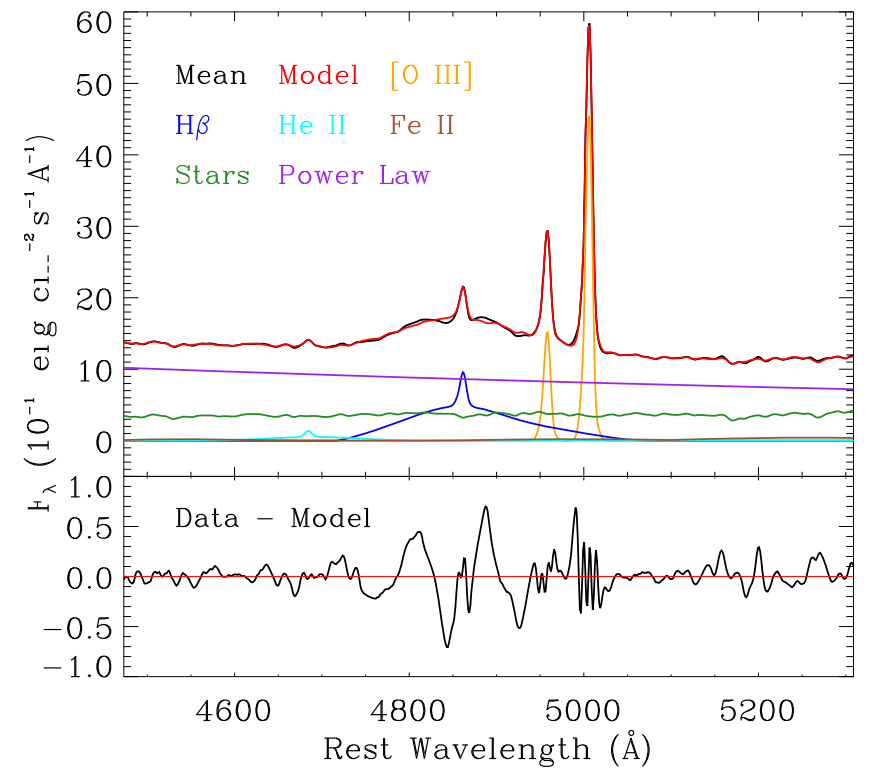

Figure 4. Top: spectral decomposition components of the mean MDM spectrum. The red spectrum is the sum of all model components and traces the data (black spectrum) well over most of the spectral range. Bottom: residuals from the full model fit.

The red spectrum in Figure 2 shows the rms of the MDM spectra after subtracting the AGN continuum and stellar continuum models from individual spectra so that only the emission-line components remain. This rms spectrum is expected to be a more accurate representation of the emission-line variability than the rms of the full spectra (Barth et al. 2015). We show the rms here and not the excess rms defined by Equation (4) because, for parts of the spectra dominated by continuum emission, the continuum-subtracted flux could be lower than the total flux uncertainties and the e-rms would be undefined. Thus, we use the excess rms only for the full spectrum and not for individual fit components.

Panels (a) and (c) of Figure 5 show the $\mathrm{H} \beta$ mean and rms fluxes as a function of line-of-sight velocity $\left(v_{r}\right)$ after spectral decomposition, and panel (b) shows the difference between the $\mathrm{T} 1$ and T2 mean fluxes. The rms flux has a statistical uncertainty of $\sim 12 \%$ and the [O III] residuals are much lower compared to the case with no spectral decomposition (Figure 2). The rms profile still has jagged features, which likely reflect real variability across the broad emission line.

Figure 6 shows the $1158 \AA$ UV continuum light curve from Paper I, the MDM optical $5100 \AA$ continuum light curve, the $V$ band photometric light curve from Paper III, and the MDM $\mathrm{H} \beta$ and He II $\lambda 4686$ emission-line light curves. The He II light curve reaches a flat-bottomed minimum near THJD $=6720$. This is because the He II flux includes contributions from the broad- and narrow-line components, so when the broad-line flux is near zero, the total He II line flux stays at a minimum value equal to the narrow-line flux. Light-curve statistics that quantify the variability of NGC 5548 during the monitoring period are given in Table 3. $F_{\text {var }}$ is as defined in Equation (3), and $R_{\max }$ is the ratio between the maximum and minimum fluxes.

\subsection{Host-galaxy Flux Removal}

We measured the host-galaxy contribution to the continuum using an "AGN-free" image of NGC 5548 generated by Bentz et al. (2013) after performing two-dimensional surface brightness

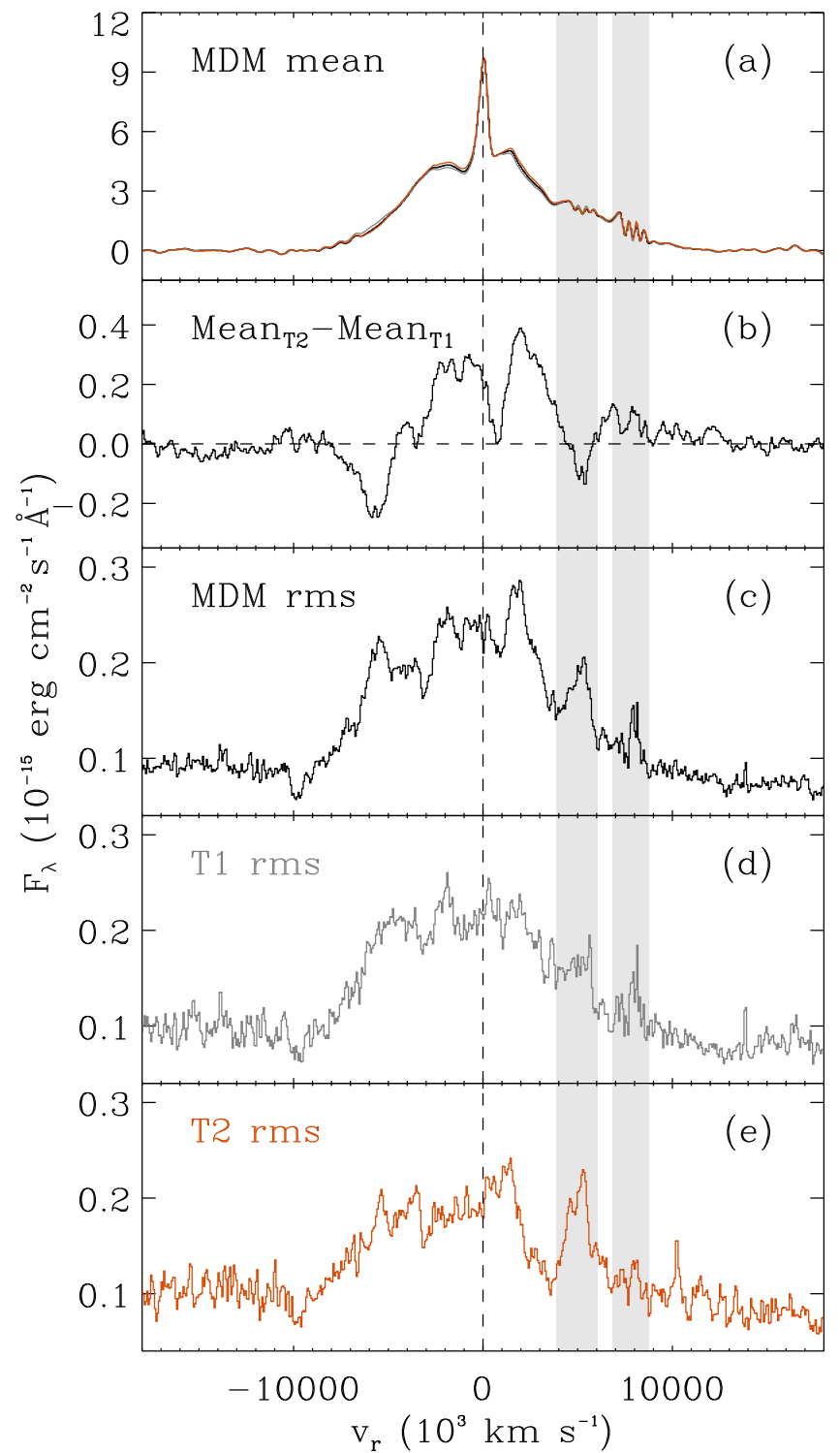

Figure 5. (a) MDM mean spectrum, (b) difference between the T1 (THJD < 6747) and T2 (THJD > 6747) mean spectra, and (c-e) rms spectra; for $\mathrm{H} \beta$ after subtracting all other fit components from spectral decomposition. The colors are for the full campaign (black), T1 (gray), and T2 (orange; see Section 3.3). Zero velocity is determined by the peak of the narrow $\mathrm{H} \beta$ line in the mean spectrum, and the gray bands indicate regions contaminated by [O III] residuals. The rms spectra have statistical uncertainties of $\sim 12 \%$.

decomposition on HST images of the galaxy. We found that the amount of starlight expected through a $5^{\prime \prime} \times 15^{\prime \prime}$ aperture with a slit position angle of $0^{\circ}$ is $F_{5100 \text {,gal }}=(4.52 \pm 0.45) \times 10^{-15} \mathrm{erg}$ $\mathrm{s}^{-1} \mathrm{~cm}^{-2} \AA^{-1}$. Subtracting this from the mean continuum flux density of $F_{5100}=(11.96 \pm 0.07) \times 10^{-15} \mathrm{erg} \mathrm{s}^{-1} \mathrm{~cm}^{-2} \AA^{-1}$ gives a mean AGN flux of $F_{5100, \mathrm{AGN}}=(7.44 \pm 0.50) \times 10^{-15}$ erg s $\mathrm{cm}^{-2} \AA^{-1}$, which is consistent with the value of $F_{5100}$, $\mathrm{AGN}=(7.82 \pm 0.02) \times 10^{-15} \mathrm{erg} \mathrm{s}^{-1} \mathrm{~cm}^{-2} \AA^{-1}$ measured from the power-law component of the spectral decomposition for the mean MDM spectrum.

\subsection{Anomalous Emission-line Light-curve Behavior}

RM analyses typically assume that the emission-line light curve responds linearly to continuum variations and is a lagged, 


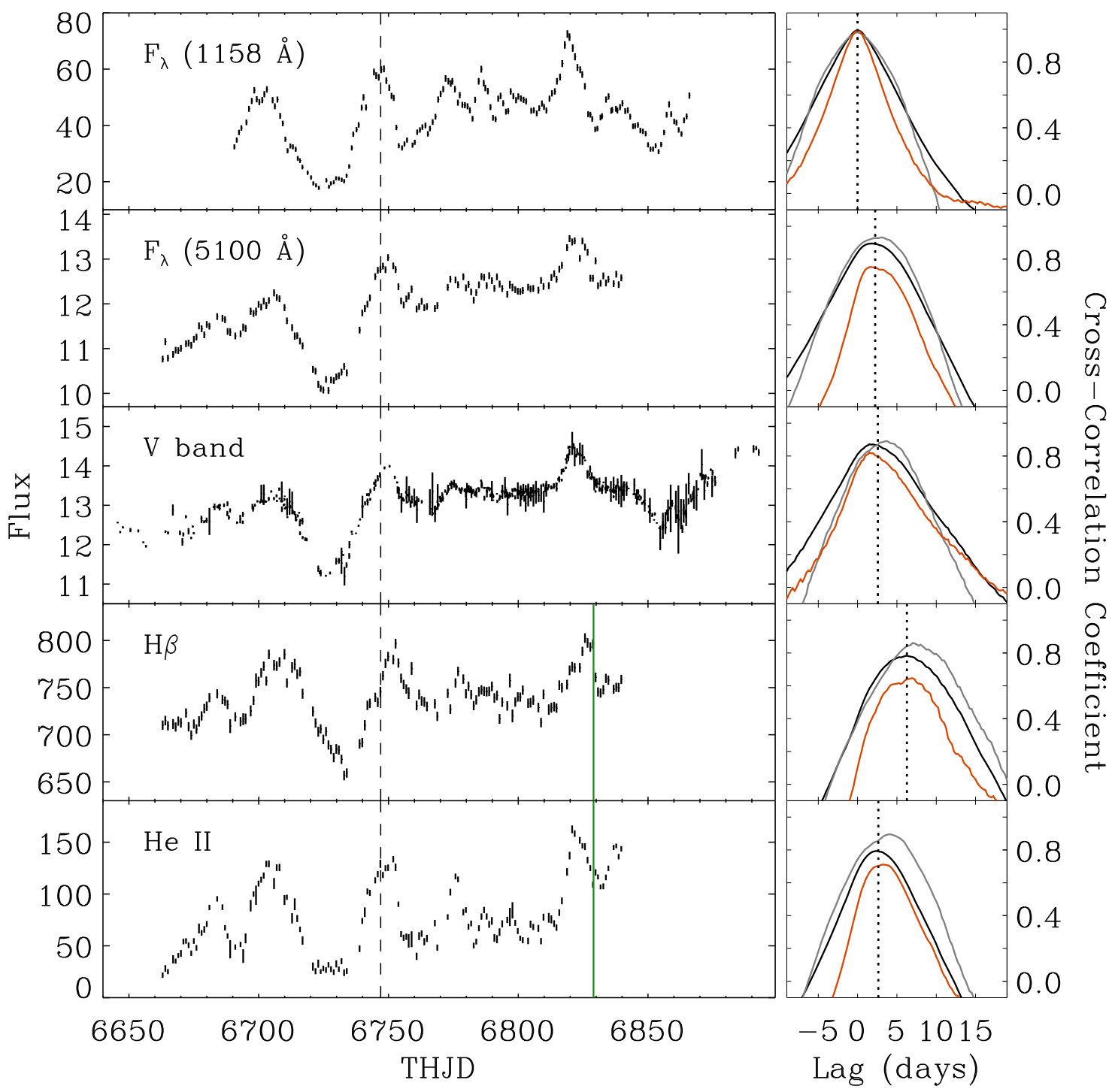

Figure 6. Left: light curves for the UV $1158 \AA$ continuum, optical $5100 \AA$ continuum, $V$-band continuum, $\mathrm{H} \beta$, and He II $\lambda 4686$. The continuum light curves are in units of $10^{-15} \mathrm{erg} \mathrm{s}^{-1} \mathrm{~cm}^{-2} \AA^{-1}$, and the line light curves are in units of $10^{-15} \mathrm{erg} \mathrm{s}^{-1} \mathrm{~cm}^{-2}$. The $\mathrm{H} \beta$ and He II fluxes include contributions from both broad- and narrow-line components. The solid green vertical line indicates where the emission-line light curves were truncated for the lag analysis (see text), and the dashed black vertical line shows the division between the T1 and T2 periods. Right: cross-correlation functions for each light curve measured against the $1158 \AA$ continuum. The top right panel shows the autocorrelation of the $1158 \AA$ light curve. The black, gray, and orange solid lines represent the CCFs for the full campaign, T1, and T2, respectively, and the dotted vertical lines denote $\tau_{\text {cen }}$ for the full campaign.

scaled, and smoothed version of the continuum light curve. However, this does not appear to be the case for a portion of our campaign. As described in Papers I and IV, there are significant differences between the UV continuum and emission-line light curves after THJD $=6780$. The continuum flux increased while the emission-line fluxes either decreased or remained roughly constant in a suppressed state for the remainder of the campaign.

The $\mathrm{H} \beta$ emission line shows similar behavior to that of $\operatorname{Ly} \alpha$ and $\mathrm{CIV}$, in that there is a marked difference in the line response between the first and second halves of the campaign. This "decorrelation" phenomenon is illustrated in Figure 7(a), where the $\mathrm{H} \beta$ and UV continuum light curves trace each other well in the first half of the campaign, but the continuum flux continues to trend upward beyond THJD $=6740$, while the $\mathrm{H} \beta$ flux begins to fall. For the remainder of the campaign, the $\mathrm{H} \beta$ flux remains in a suppressed state and the light curve does not follow the continuum light curve well in that prominent features in the continuum light curves (e.g., THJD $\approx 6770$, $6785)$ are not present in the emission-line light curve. The He II $\lambda 4686$ light curve behaves similarly and decorrelates from the continuum at around THJD $=6760$ (Figure 7(b)). This phenomenon is also apparent when comparing the $\mathrm{H} \beta$ light curve to the $5100 \AA$ continuum, as shown in Figure 7 (c). Though there are small differences between the light curves in $\mathrm{T} 1$ (such as around THJD 6685), the overall correlation in T1 is significantly better than in $\mathrm{T} 2$.

Owing to this change in the emission-line response, we followed the procedures presented in Paper I for determining the UV emission line lags and divided the $5100 \AA$ continuum and optical emission-line light curves into two subsets, labeled $\mathrm{T} 1$ and $\mathrm{T} 2$, to examine the lag of each segment separately. The subsets are divided at THJD $=6747$, and each has 67 epochs. The $1158 \AA$ and $V$-band continuum light curves were also 
Table 3

Statistics for $H S T$ and MDM Light Curves

\begin{tabular}{|c|c|c|c|c|c|}
\hline Emission Component & Epochs & Mean Flux & rms Flux & $F_{\mathrm{var}}$ & $R_{\max }$ \\
\hline$\overline{F_{\lambda}(1158 \AA)}$ & 171 & $43.48 \pm 0.86$ & $11.14 \pm 1.21$ & 0.255 & $4.07 \pm 0.18$ \\
\hline$F_{\lambda}(5100 \AA)$ & 133 & $11.96 \pm 0.07$ & $0.80 \pm 0.09$ & 0.066 & $1.33 \pm 0.01$ \\
\hline $\mathrm{H} \beta$ & 133 & $738.49 \pm 2.40$ & $28.29 \pm 3.38$ & 0.038 & $1.22 \pm 0.01$ \\
\hline He II $\lambda 4686$ & 133 & $78.71 \pm 2.95$ & $35.14 \pm 4.17$ & 0.444 & $7.48 \pm 0.91$ \\
\hline$F_{\lambda}(1158 \AA, \mathrm{T} 1)$ & 51 & $35.85 \pm 1.79$ & $12.61 \pm 2.54$ & 0.351 & $3.31 \pm 0.15$ \\
\hline$F_{\lambda}(1158 \AA 0, \mathrm{~T} 2)$ & 120 & $46.72 \pm 0.80$ & $8.66 \pm 1.13$ & 0.184 & $2.36 \pm 0.08$ \\
\hline$F_{\lambda}(5100 \AA, \mathrm{T} 1)$ & 67 & $11.31 \pm 0.08$ & $0.67 \pm 0.12$ & 0.059 & $1.27 \pm 0.01$ \\
\hline$F_{\lambda}(5100 \AA, \mathrm{T} 2)$ & 67 & $12.51 \pm 0.04$ & $0.37 \pm 0.06$ & 0.029 & $1.13 \pm 0.01$ \\
\hline $\mathrm{H} \beta(\mathrm{T} 1)$ & 67 & $725.10 \pm 3.80$ & $30.47 \pm 5.36$ & 0.041 & $1.20 \pm 0.01$ \\
\hline $\mathrm{H} \beta(\mathrm{T} 2)$ & 67 & $750.14 \pm 2.38$ & $20.11 \pm 3.33$ & 0.026 & $1.13 \pm 0.01$ \\
\hline He II $\lambda 4686$ (T1) & 67 & $65.84 \pm 4.16$ & $33.61 \pm 5.89$ & 0.507 & $5.95 \pm 0.73$ \\
\hline He II $\lambda 4686$ (T2) & 67 & $89.90 \pm 3.75$ & $32.71 \pm 5.34$ & 0.362 & $4.07 \pm 0.37$ \\
\hline
\end{tabular}

Note. Continuum flux densities are in units of $10^{-15} \mathrm{erg} \mathrm{s}^{-1} \mathrm{~cm}^{-2} \AA^{-1}$, and emission-line fluxes are in units of $10^{-15} \mathrm{erg} \mathrm{s}^{-1} \mathrm{~cm}^{-2}$. T1 and T2 denote the first and second halves of the campaign (respectively) divided at THJD $=6747$.

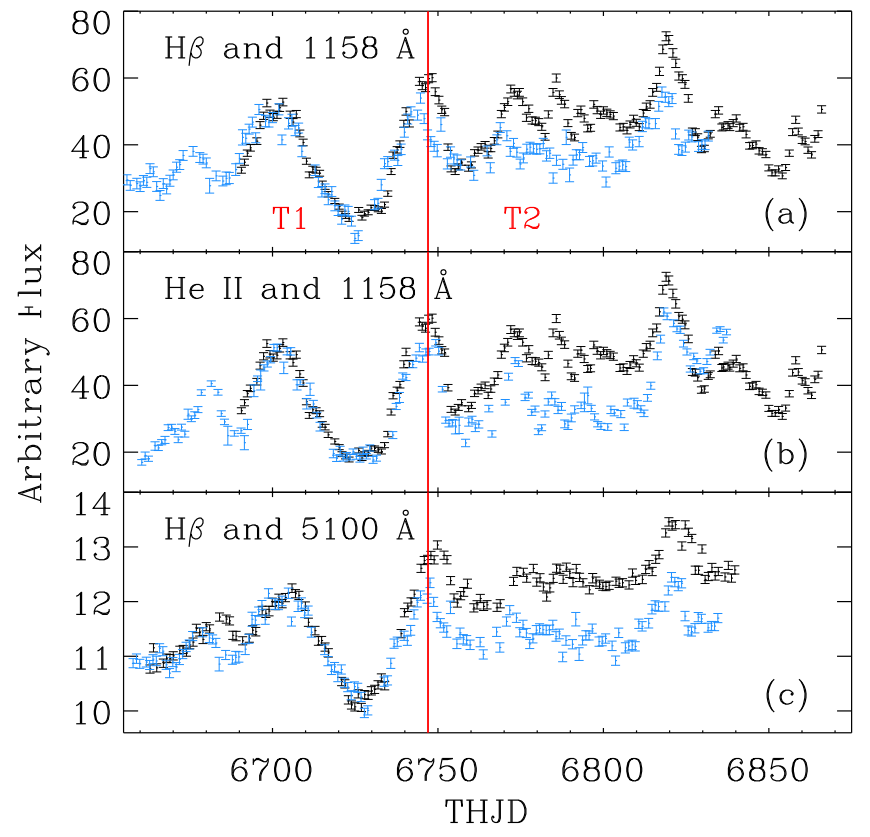

Figure 7. $1158 \AA$ and $5100 \AA$ continuum light curves (black) compared with scaled and shifted emission-line light curves (blue). The vertical line at THJD $=6747$ indicates the epoch separating the T1 and T2 segments. In each of the panels, the emission-line light curve closely tracks the continuum light curve in T1, but appears to correlate less closely with the continuum variations in $\mathrm{T} 2$.

separated into two segments at THJD $=6747$. Note that this is a different dividing epoch from the one used in Paper I. The characteristics of the half-campaign light curves are given in the bottom portion of Table 3. Figure 5 shows the MDM mean and rms spectra for $\mathrm{T} 1$ and $\mathrm{T} 2$ in gray and orange, respectively. While the three mean spectra look almost identical, the T1 and T2 rms spectra are significantly different, which indicates changes in the amount of variability between the two campaign halves.

\section{Data Analysis}

In the following sections, we examine properties of the BLR by measuring the emission-line responses to continuum variations. We also discuss the anomalous behavior of the emission-line light curves observed during this campaign and $\mathrm{BH}$ mass measurements using this data set.

\subsection{Emission-line Lags}

We measured the $\mathrm{H} \beta$ and $\mathrm{He}$ II $\lambda 4686$ lags relative to both the $5100 \AA$ continuum and the $1158 \AA$ continuum. All light curves were detrended by subtracting a linear least-squares fit to the data to remove long-term trends that may bias lag calculations (Welsh 1999). In this case, we found very weak trends for all the light curves, and detrending has a very small effect ( $\sim 0.01$ days) on the measured lags. We computed the cross-correlation coefficient $r$ for lags between -20 and 40 days in increments of 0.25 days using the interpolated crosscorrelation function (ICCF; White \& Peterson 1994). Two lag estimates were made for each light-curve pair-the value corresponding to $r_{\max }\left(\tau_{\text {peak }}\right)$ and the centroid of all values with $r>0.8 r_{\text {max }}\left(\tau_{\text {cen }}\right)$. Estimates for the final $\tau_{\text {peak }}$ and $\tau_{\text {cen }}$ values and their uncertainties were obtained using Monte Carlo bootstrapping analysis (Peterson et al. 2004), where many realizations of the continuum and emission-line light curves were created by randomly choosing $n$ data points with replacement from the observed light curves, where $n$ is the total number of points in the data set. If a data point is picked $m$ times, then the uncertainty on that point is decreased by a factor of $m^{1 / 2}$. Each value is then varied by a random Gaussian deviate scaled by the measured flux uncertainty. We constructed $10^{3}$ realizations of each light curve and computed the cross-correlation function (CCF) for each pair of line and continuum light-curve realizations to create a distribution of $\tau_{\text {peak }}$ and $\tau_{\text {cen }}$ values. The median value from each distribution and the central $68 \%$ interval are then taken to be the final lag and its uncertainty.

Table 4 lists the ICCF lags for $\mathrm{H} \beta$ and He II $\lambda 4686$ measured against the $1158 \AA, 5100 \AA$, and $V$-band continua. The lag between the $5100 \AA$ and $1158 \AA$ continua is also given. For comparison with Paper I, which presents the UV emission-line lags against the $1367 \AA$ continuum, we also include $\mathrm{H} \beta$ and He II lags measured against this continuum. Distributions of $\tau_{\text {cen }}$ values from the Monte Carlo bootstrap analysis using the $1158 \AA$ continuum are shown in the top panels of Figure 8 . 
Table 4

Rest-frame Emission-line Lags

\begin{tabular}{|c|c|c|c|c|c|c|c|}
\hline Light Curves & $\tau_{\text {peak }}$ & $\tau_{\text {cen }}$ & $\tau_{\text {cen,T1 }}$ & $\tau_{\text {cen, } \mathrm{T} 2}$ & $\tau_{\text {JAVELIN }}$ & $\tau_{\text {JAVELIN }, \mathrm{T} 1}$ & $\tau_{\text {JAVELIN }, \mathbf{T} 2}$ \\
\hline $\mathrm{H} \beta$ versus $F_{\lambda}(1158 \AA)$ & $6.14_{-0.98}^{+0.74}$ & $6.23_{-0.44}^{+0.39}$ & $7.62_{-0.49}^{+0.49}$ & $5.99_{-0.75}^{+0.71}$ & $6.56_{-0.49}^{+0.48}$ & $6.91_{-0.63}^{+0.64}$ & $7.42_{-1.07}^{+0.97}$ \\
\hline $\mathrm{H} \beta$ versus $F_{\lambda}(1367 \AA)$ & $5.90_{-0.74}^{+0.25}$ & $5.89_{-0.37}^{+0.37}$ & $7.24_{-0.48}^{+0.49}$ & $5.99_{-0.82}^{+0.76}$ & $6.12_{-0.47}^{+0.46}$ & $6.52_{-0.57}^{+0.60}$ & $7.11_{-1.06}^{+1.03}$ \\
\hline $\mathrm{H} \beta$ versus $V$ band & $3.93_{-0.98}^{+0.98}$ & $3.79_{-0.34}^{+0.37}$ & $3.82_{-0.47}^{+0.57}$ & $4.13_{-0.58}^{+0.55}$ & $3.54_{-0.46}^{+0.45}$ & $4.89_{-0.71}^{+0.66}$ & $4.05_{-0.78}^{+0.93}$ \\
\hline He II versus $F_{\lambda}(1158 \AA)$ & $2.46_{-0.25}^{+0.49}$ & $2.69_{-0.25}^{+0.24}$ & $3.71_{-0.38}^{+0.39}$ & $3.19_{-0.35}^{+0.36}$ & $2.65_{-0.27}^{+0.27}$ & $3.27_{-0.35}^{+0.35}$ & $2.99_{-0.26}^{+0.25}$ \\
\hline He II versus $F_{\lambda}(1367 \AA)$ & $2.21_{-0.25}^{+0.25}$ & $2.45_{-0.24}^{+0.25}$ & $3.43_{-0.43}^{+0.36}$ & $3.16_{-0.33}^{+0.29}$ & $2.41_{-0.26}^{+0.25}$ & $3.04_{-0.36}^{+0.35}$ & $2.79_{-0.25}^{+0.25}$ \\
\hline$F_{\lambda}(5100 \AA)$ versus $F_{\lambda}(1158 \AA)$ & $1.97_{-0.49}^{+0.25}$ & $2.23_{-0.26}^{+0.31}$ & $2.55_{-0.33}^{+0.28}$ & $2.77_{-0.45}^{+0.41}$ & $\ldots$ & $\ldots$ & $\ldots$ \\
\hline $\mathrm{H} \beta_{\text {full,MDM }}$ versus $F_{\lambda}(1158 \AA)$ & $5.90_{-0.49}^{+0.74}$ & $6.26_{-0.37}^{+0.38}$ & $\ldots$ & $\ldots$ & $\cdots$ & $\cdots$ & $\cdots$ \\
\hline $\mathrm{H} \beta_{\text {full,allsites }}$ versus $F_{\lambda}(1158 \AA)$ & $5.65_{-0.25}^{+0.74}$ & $6.49_{-0.37}^{+0.37}$ & $\ldots$ & $\cdots$ & $\ldots$ & $\ldots$ & $\ldots$ \\
\hline
\end{tabular}

Note. Rest-frame $\mathrm{H} \beta$ and He II $\lambda 4686$ lags (days) for the full campaign and for the T1 (THJD < 6747) and T2 (THJD > 6747) subsets, measured using both ICCF and JAVELIN. The last two lines show the $\mathrm{H} \beta$ lags measured using the light curve derived without spectral decomposition up to THJD $=6828.75$.

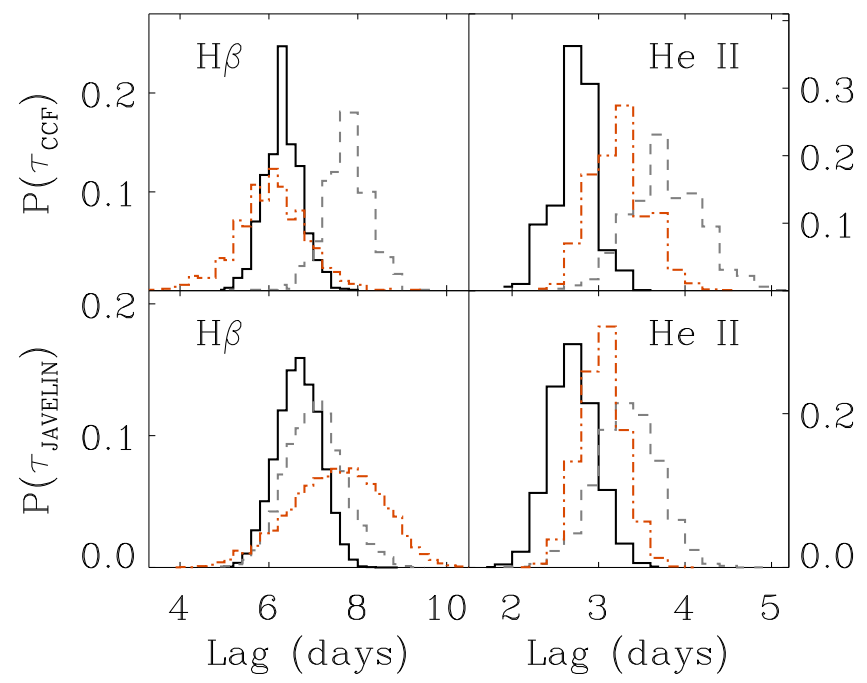

Figure 8. $\tau_{\text {cen }}$ (top) and JAVELIN (bottom) lag probability distributions for $\mathrm{H} \beta$ (left) and He II (right) measured against the $1158 \AA$ continuum. Black solid lines are for the full campaign, gray dashed lines are for $\mathrm{T} 1$, and orange dotdashed lines are for $\mathrm{T} 2$.

We also computed $\mathrm{H} \beta$ and $\mathrm{He}$ II $\lambda 4686$ lags for time periods $\mathrm{T} 1$ and $\mathrm{T} 2$ and found the $\mathrm{T} 1$ lags to be consistently longer by about $2 \sigma$. For $\mathrm{H} \beta$, the $\mathrm{T} 1$ and $\mathrm{T} 2$ lags bracket the full-campaign lag, while for He II $\lambda 4686$, the full-campaign lag is shorter than both T1 and T2 lags. For comparison, Paper I found the Ly $\alpha$, Si IV, C IV, and He II $\lambda 1640$ lags to be longer for T2 than for $\mathrm{T} 1$, which is the opposite of what we find for $\mathrm{H} \beta$.

To illustrate the effects of spectral decomposition, we also include in Table 4 the ICCF lags for the $\mathrm{H} \beta$ light curve where the fluxes were measured using the straight-line continuumsubtraction method and without spectral decomposition. We calculated lags for both the MDM-only and the multisite $\mathrm{H} \beta$ light curves, which were truncated at THJD $=6828.75$ to exclude the last 10 epochs of MDM data (see Section 2). The lags measured with and without using spectral decomposition are consistent to within $1 \sigma$.

In addition to the ICCF method, we computed the emissionline lags using the JAVELIN suite of Python codes ( $\mathrm{Zu}$ et al. 2011). We used JAVELIN to linearly detrend the light curves and model the AGN continuum variability as a damped

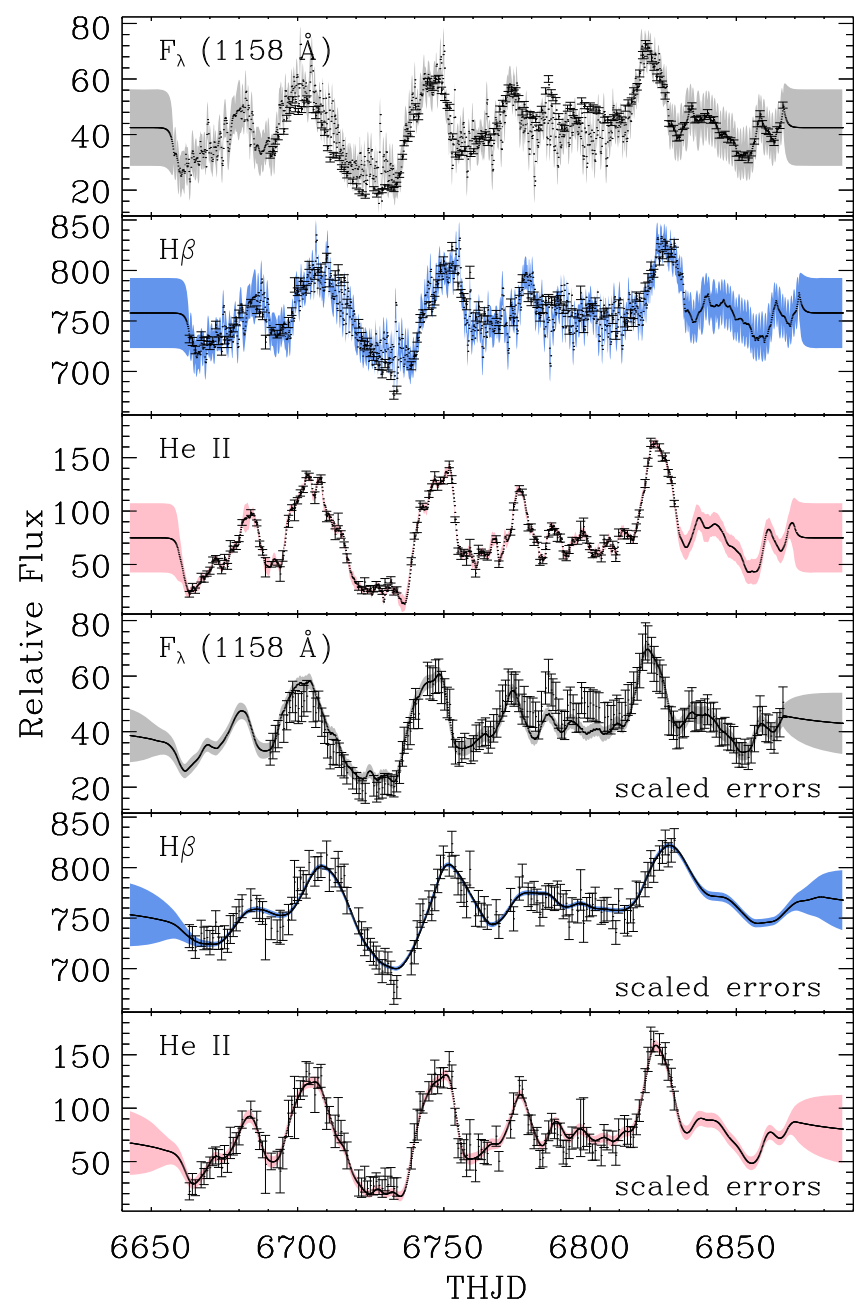

Figure 9. JAVELIN light curves from simultaneously modeling the $\mathrm{H} \beta$ and He II $\lambda 4686$ emission lines with the UV $1158 \AA$ continuum. The data points are measured from observations, the black solid lines are the weighted means of the model light curves consistent with the data, and the thickness of the shaded regions indicates the $1 \sigma$ spread of those light curves. The top three panels show the observed data and JAVELIN model light curves without any error scaling, and the bottom three panels show the data and light-curve models with scaled uncertainties.

random walk process (DRW; Kelly et al. 2009; Zu et al. 2013). JAVELIN explicitly models the emission-line light curves as smoothed, scaled, and lagged versions of the continuum light 


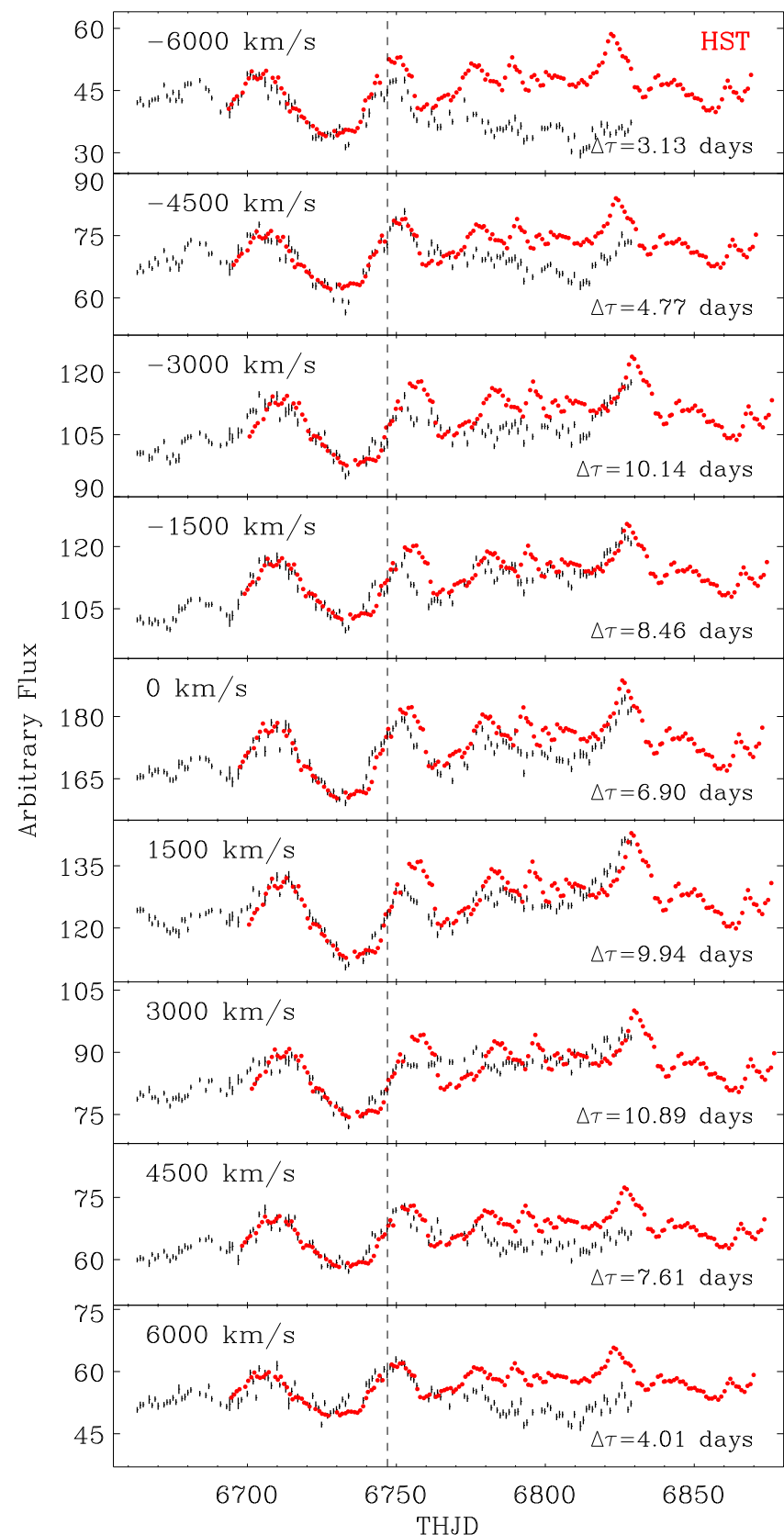

Figure 10. Velocity-resolved $\mathrm{H} \beta$ light curves for $1500 \mathrm{~km} \mathrm{~s}^{-1}$ bins (black) with the central velocity for each bin shown at the top left of each panel. HST $1158 \AA$ light curves that have been scaled and shifted to match the first half of the $\mathrm{H} \beta$ light curves are shown in red, and the dashed line indicates the epoch that separates $\mathrm{T} 1$ and $\mathrm{T} 2$ for this analysis. The $\mathrm{H} \beta$ response to the continuum is distinctly velocity dependent during the second half of the campaign.

curve. Since the decorrelation of the line and continuum light curves during the latter half of the campaign clearly violates these assumptions, it is of interest to examine the consequences for the JAVELIN models. For these models, we simultaneously fit the $\mathrm{H} \beta$ and $\mathrm{He}$ II light curves using either the $1158 \AA$, $5100 \AA$, or $V$-band continuum light curve. The AGN STORM light curves are too short to accurately determine the DRW damping timescale, so this value was fixed to $\tau_{\text {DRW }} \approx 164$ days as derived by $\mathrm{Zu}$ et al. (2011) from fits to the $13 \mathrm{yr}$ light curve of NGC 5548 (Peterson et al. 2002). The precise value of $\tau_{\text {DRW }}$ is not critical for the algorithm to work provided that it is approximately correct.
The top three panels of Figure 9 show the results of using JAVELIN directly on the observed data. Despite the long DRW timescale, the light-curve models show rapid fluctuations. The algorithm tries to match the suppressed flux in the line light curve to the continuum light curve, and because of the small uncertainties, it strongly prefers lag times for which the continuum and line light curves have minimal temporal overlap. This caused the posterior lag distribution to have multiple narrow peaks corresponding to lags that best desynchronize the light curves.

We can attempt to compensate for this problem by increasing the uncertainties to encompass the amplitude of the decorrelation. This requires scaling up the full-campaign light-curve uncertainties by factors of 5 and 3 for the continuum and line light curves, respectively. The lower panels in Figure 9 show the JAVELIN results from fitting to the light curves with scaled errors, where the broader uncertainties allow the algorithm to construct smooth light-curve models. Since there is more statistical weight from fitting both lines simultaneously, the models track the line light curves best and show a smooth systematic offset for the continuum where the line and continuum light curves are decorrelated. When computing the half-campaign lags, JAVELIN favors a smaller line flux scale factor for $\mathrm{T} 2$ to account for the suppressed line fluxes, which begins near the epoch separating $\mathrm{T} 1$ and $\mathrm{T} 2$. We therefore did not need to scale the flux errors by as much as for the full campaign to account for the decorrelation, and we used an error scaling factor of 3 for both continuum and line light curves. The resulting JAVELIN lags, shown in Table 4, are consistent with those measured using the ICCF method. Similar to the ICCF lags, the JAVELIN lags for the full-campaign light curves are also shorter than those for T1 and T2. However, since the JAVELIN assumptions of the relationship between the line and continuum light curves are not valid for this campaign and the flux errors were scaled for the sole purpose of producing convergent solutions, we do not use the JAVELIN lags for subsequent analysis.

We examined the velocity-resolved emission-line response by dividing the $\mathrm{H} \beta$ line profile into bins with velocity width of $500 \mathrm{~km} \mathrm{~s}^{-1}$ and set zero velocity using the peak of the narrow $\mathrm{H} \beta$ component in the mean spectrum. We constructed light curves for each velocity bin separately, and Figure 10 shows the light curves for $1500 \mathrm{~km} \mathrm{~s}^{-1}$ velocity bins across the $\mathrm{H} \beta$ line profile (black), with the velocity at the center of each bin shown in the top left of each panel. There were six epochs of spectra $^{94}$ that produced outlying $\mathrm{H} \beta$ fluxes and significantly higher than average flux uncertainties for individual velocity bins, so we removed them from the velocity-resolved light curves in order to improve the lag measurements.

We determined the ICCF lag for each of these binned light curves with respect to both the UV and optical continua, and we show these lags as a function of line-of-sight velocity in the top left panel of Figure 11. The middle left panel shows the velocity-resolved $\mathrm{H} \beta-\mathrm{UV}$ lag for $\mathrm{T} 1$ and $\mathrm{T} 2$, and the maximum cross-correlation coefficients $\left(r_{\max }\right)$ are shown in the right panels. The bottom panel of Figure 11 shows the MDM fullcampaign mean spectrum for reference, and Table 5 lists the velocity-resolved lags. Emission-line variations have lower amplitudes during T2 compared with T1, which led to poorly constrained ICCF lags with large uncertainties for velocity

$\overline{{ }^{94} \mathrm{THJD}}=[6689.0,6699.0,6757.8,6758.9,6796.8,6797.8]$. 


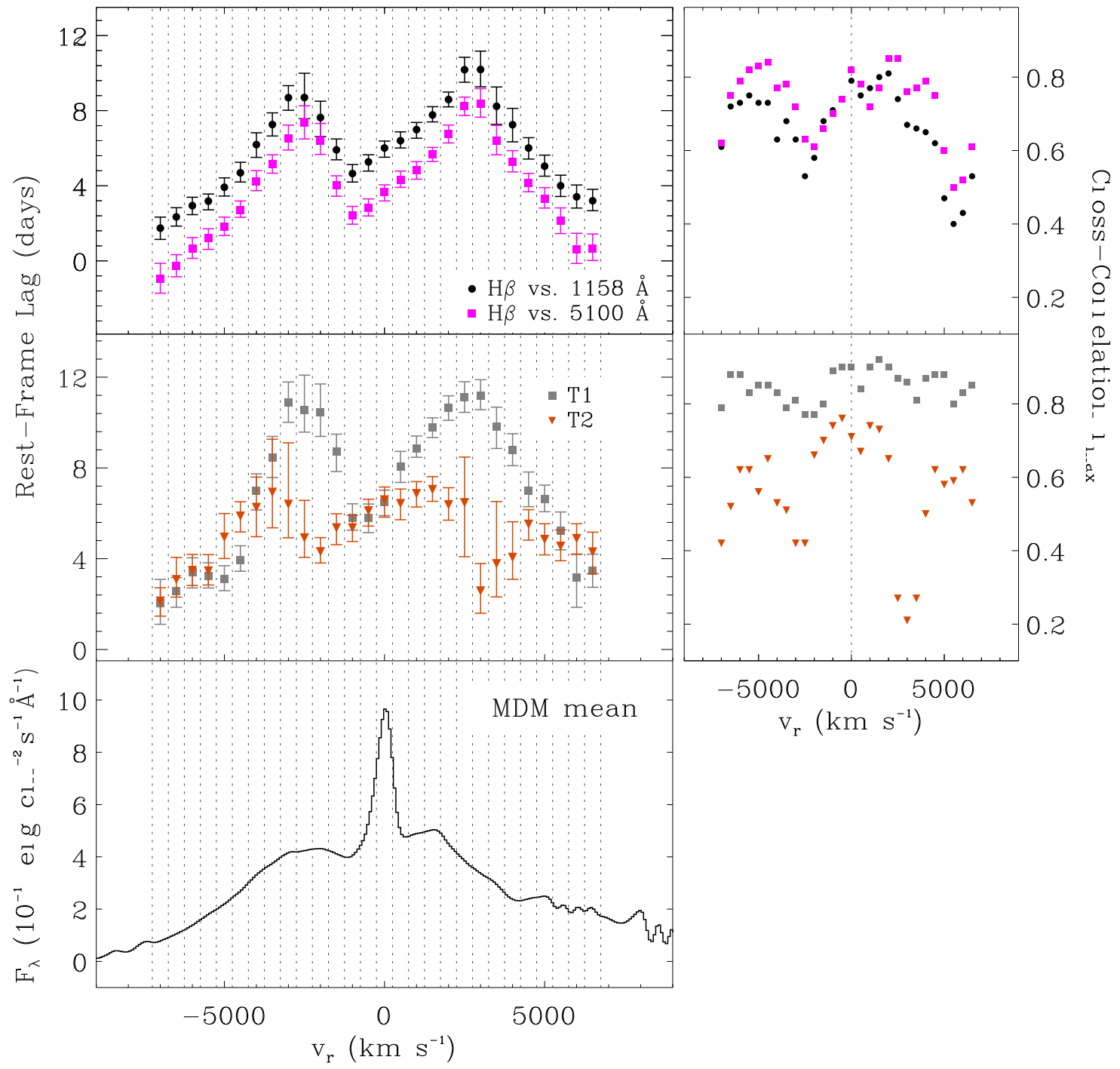

Figure 11. Top left: ICCF H $\beta$ lags $\left(\tau_{\text {cen }}\right)$ for $500 \mathrm{~km} \mathrm{~s}^{-1}$ bins measured against the $1158 \AA$ and $5100 \AA$ continua. Middle left: lags for T1 and T2 measured against the $1158 \AA$ continuum. Bottom left: MDM mean spectrum for the full campaign. Right: maximum cross-correlation coefficients $\left(r_{\text {max }}\right)$ for individual velocity bins.

bins with low line flux. We therefore reduced the upper limit of the CCF lag range from 40 to 20 days when computing the $\mathrm{T} 2$ lags.

The velocity-resolved $\mathrm{H} \beta-\mathrm{UV}$ lag for the full campaign is shortest $\left(\tau_{\text {cen }} \approx 2\right.$ days $)$ in the line wings where $v_{r} \approx \pm 7000 \mathrm{~km} \mathrm{~s}^{-1}$. The lag increases as $v_{r}$ approaches zero from both sides of the lag profile, reaches local maxima of $\tau_{\text {cen }} \approx 10$ days at about $v_{r} \approx \pm 3000 \mathrm{~km} \mathrm{~s}^{-1}$, and then steadily decreases until it reaches a local minimum of $\tau_{\text {cen }} \approx 4$ days near the line profile center. The lag profile measured against the optical continuum has a similar shape, but with all lags $\sim 2-3$ days shorter, as we would expect from the $\sim 2$-day lag between the two continua (Table 4). A similar double-peaked lag profile is also observed for $\operatorname{Ly} \alpha$ (see Paper I). The T1 lag profile closely resembles that of the full campaign, but the T2 lag profile shows a slightly different structure. The bins where the $\mathrm{T} 1$ and T2 lags are most discrepant are also where the T2 light curves are least correlated with the continuum $\left(r_{\max }<0.4\right)$. However, even excluding these outliers, there are still discernible differences between the $\mathrm{T} 1$ and $\mathrm{T} 2$ lag profiles.
Additionally, the $\mathrm{T} 2 r_{\max }$ values are lower than those of $\mathrm{T} 1 \mathrm{in}$ every velocity bin, which clearly demonstrates that the line and continuum light curves are less correlated in $\mathrm{T} 2$ than in $\mathrm{T} 1$.

The shape of the velocity-resolved lag profile can provide qualitative information about the kinematics of the lineemitting gas (e.g., Kollatschny 2003; Bentz et al. 2009b; Denney et al. 2010; Barth et al. 2011; Du et al. 2016a). In simple models of the BLR (Ulrich et al. 1984; Gaskell 1988; Welsh \& Horne 1991; Horne et al. 2004; Goad et al. 2012; Gaskell \& Goosmann 2013; Grier et al. 2013), pure infall motion would lead to longer lags on the blue side of the line profile, and for outflow, the most redshifted gas would have the longest lag. For gas in Keplerian orbits, the shortest lags would be in the line wings, since gas with higher $v_{r}$ is closer to the central BH. Gas with very low $v_{r}$ could have a wide range of lags, and a spherical or flat disk distribution of BLR clouds in Keplerian motion could lead to a double-peaked velocityresolved lag profile if the ionizing source is emitting anisotropically (Welsh \& Horne 1991; Goad \& Wanders 1996; Horne et al. 2004). 
Table 5

Rest-frame $\mathrm{H} \beta$ Velocity-resolved Lags

\begin{tabular}{|c|c|c|c|c|c|c|c|}
\hline $\begin{array}{l}\text { Wavelength } \\
(\AA)\end{array}$ & $\begin{array}{c}v_{r} \\
\left(\mathrm{~km} \mathrm{~s}^{-1}\right)\end{array}$ & $\begin{array}{r}\tau_{\text {Full }} \\
\text { (days) }\end{array}$ & $r_{\text {max,Full }}$ & $\begin{array}{r}\tau_{\mathrm{T} 1} \\
\text { (days) }\end{array}$ & $r_{\max , \mathrm{T} 1}$ & $\begin{array}{r}\tau_{\mathrm{T} 2} \\
\text { (days) }\end{array}$ & $r_{\max , \mathrm{T} 2}$ \\
\hline $4743.82-4751.92$ & 7000 & $1.74_{-0.60}^{+0.59}$ & 0.61 & $2.05_{-0.94}^{+1.04}$ & 0.79 & $2.13_{-0.66}^{+0.59}$ & 0.42 \\
\hline $4751.92-4760.03$ & 6500 & $2.34_{-0.49}^{+0.49}$ & 0.72 & $2.57_{-0.71}^{+0.60}$ & 0.88 & $3.08_{-0.77}^{+0.98}$ & 0.52 \\
\hline $4760.03-4768.13$ & 6000 & $2.94_{-0.48}^{+0.45}$ & 0.73 & $3.41_{-0.70}^{+0.63}$ & 0.88 & $3.48_{-0.66}^{+0.71}$ & 0.62 \\
\hline $4768.13-4776.23$ & 5500 & $3.18_{-0.46}^{+0.38}$ & 0.75 & $3.25_{-0.54}^{+0.57}$ & 0.83 & $3.46_{-0.62}^{+0.72}$ & 0.62 \\
\hline $4776.23-4784.33$ & 5000 & $3.92_{-0.47}^{+0.50}$ & 0.73 & $3.12_{-0.53}^{+0.57}$ & 0.85 & $4.95_{-0.94}^{+1.04}$ & 0.56 \\
\hline $4800.53-4808.63$ & 3500 & $7.26_{-0.61}^{+0.62}$ & 0.68 & $8.45_{-0.87}^{+0.94}$ & 0.79 & $6.93_{-1.57}^{+2.34}$ & 0.51 \\
\hline $4808.63-4816.73$ & 3000 & $8.69_{-0.67}^{+0.64}$ & 0.63 & $10.90_{-0.89}^{+0.89}$ & 0.81 & $6.40_{-1.48}^{+2.71}$ & 0.42 \\
\hline $4816.73-4824.84$ & 2500 & $8.70_{-1.13}^{+1.29}$ & 0.53 & $10.54_{-0.96}^{+1.55}$ & 0.77 & $4.92_{-0.86}^{+1.65}$ & 0.42 \\
\hline $4824.84-4832.94$ & 2000 & $7.63_{-1.01}^{+0.87}$ & 0.58 & $10.46_{-1.07}^{+1.24}$ & 0.77 & $4.31_{-0.50}^{+0.62}$ & 0.66 \\
\hline 4832.94-4841.05 & 1500 & $5.91_{-0.53}^{+0.58}$ & 0.68 & $8.71_{-0.87}^{+0.78}$ & 0.80 & $5.36_{-0.74}^{+0.62}$ & 0.70 \\
\hline $4841.05-4849.15$ & 1000 & $4.65_{-0.45}^{+0.48}$ & 0.71 & $5.79_{-0.53}^{+0.63}$ & 0.89 & $5.36_{-0.60}^{+0.55}$ & 0.74 \\
\hline $4873.45-4881.56$ & 1000 & $6.99_{-0.46}^{+0.38}$ & 0.77 & $8.86_{-0.39}^{+0.55}$ & 0.90 & $6.87_{-0.59}^{+0.53}$ & 0.74 \\
\hline $4881.56-4889.66$ & 1500 & $7.77_{-0.37}^{+0.44}$ & 0.80 & $9.80_{-0.46}^{+0.41}$ & 0.92 & $7.05_{-0.52}^{+0.57}$ & 0.73 \\
\hline $4889.66-4897.76$ & 2000 & $8.59_{-0.39}^{+0.40}$ & 0.81 & $10.65_{-0.55}^{+0.53}$ & 0.90 & $6.38_{-0.68}^{+0.75}$ & 0.65 \\
\hline $4897.76-4905.86$ & 2500 & $10.18_{-0.67}^{+0.66}$ & 0.74 & $11.13_{-0.68}^{+0.67}$ & 0.87 & $6.47_{-2.38}^{+2.01}$ & 0.27 \\
\hline 4905.86-4913.96 & 3000 & $10.19_{-0.92}^{+0.98}$ & 0.67 & $11.18_{-0.61}^{+0.71}$ & 0.86 & $2.58_{-0.98}^{+1.21}$ & 0.21 \\
\hline $4913.96-4922.06$ & 3500 & $8.23_{-0.96}^{+1.03}$ & 0.66 & $9.82_{-0.96}^{+0.86}$ & 0.81 & $3.78_{-1.46}^{+2.74}$ & 0.27 \\
\hline 4922.06-4930.16 & 4000 & $7.25_{-0.91}^{+0.87}$ & 0.65 & $8.80_{-0.70}^{+0.71}$ & 0.87 & $4.06_{-0.97}^{+1.57}$ & 0.50 \\
\hline 4930.16-4938.27 & 4500 & $6.01_{-0.59}^{+0.55}$ & 0.62 & $7.01_{-0.72}^{+0.81}$ & 0.88 & $5.52_{-0.70}^{+0.64}$ & 0.62 \\
\hline $4938.27-4946.38$ & 5000 & $5.04_{-0.53}^{+0.59}$ & 0.47 & $6.63_{-0.56}^{+0.61}$ & 0.88 & $4.85_{-0.68}^{+0.69}$ & 0.58 \\
\hline $4946.38-4954.48$ & 5500 & $4.00_{-0.57}^{+0.56}$ & 0.40 & $5.22_{-0.83}^{+0.84}$ & 0.80 & $4.55_{-0.64}^{+0.72}$ & 0.59 \\
\hline $4954.48-4962.58$ & 6000 & $3.41_{-0.60}^{+0.63}$ & 0.43 & $3.16_{-1.30}^{+1.05}$ & 0.83 & $4.89_{-0.70}^{+0.65}$ & 0.62 \\
\hline $4962.58-4970.68$ & 6500 & $3.20_{-0.53}^{+0.61}$ & 0.53 & $3.46_{-0.72}^{+0.74}$ & 0.85 & $4.30_{-0.96}^{+0.87}$ & 0.53 \\
\hline
\end{tabular}

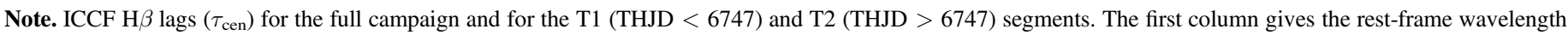

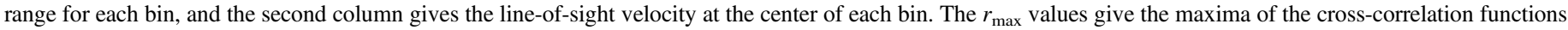
between the light curve for each velocity bin and the UV continuum.

Previous studies of the UV and optical lines in NGC 5548 have inferred either Keplerian orbits (Horne et al. 1991; Wanders et al. 1995; Denney et al. 2009; Bentz et al. 2010b) or infalling motion (Crenshaw \& Blackwell 1990; Done \& Krolik 1996; Welsh et al. 2007; Pancoast et al. 2014; Gaskell \& Goosmann 2016) for the BLR gas. From our data, the shape of the $\mathrm{H} \beta$ velocity-resolved lag profile suggests a BLR dominated by Keplerian motion. The discrepancy between the T1 and T2 lag profiles may suggest a change in the distribution or dynamics of the BLR gas, though such changes typically occur on timescales much longer than our campaign. More detailed interpretation requires comparison with transfer functions generated for various dynamical models of the BLR, which will be the subject of future work in this series (A. Pancoast et al. 2017, in preparation).

Figure 10 also shows modified versions of the $1158 \AA$ continuum light curve in red. These light curves were shifted in time by the average T1 lag of the bins incorporated in each $\mathrm{H} \beta$ light curve, which are shown in the bottom right of each panel. The fluxes were roughly scaled and shifted to match the first half of the $\mathrm{H} \beta$ light curves. Comparing the line and continuum light curves, it is evident that the $\mathrm{H} \beta$ response in $\mathrm{T} 2$ is heavily dependent on the line-of-sight velocity.

\subsection{Anomalous Emission-line Response to Continuum}

Our data show that previous assumptions about the relationship between the continuum and emission-line light curves-namely, that the emission-line light curves are smoothed, scaled, and time-shifted versions of the continuum light curve - are not always valid, as we observed all the UV and optical emission-line light curves decorrelating from the UV continuum about halfway through the monitoring period. Paper IV examined this effect for the UV emission lines by measuring changes in the emission-line equivalent width (EW),

$$
\mathrm{EW}=\frac{F_{\text {line }}}{F_{\text {cont }}},
$$

and the responsivity $\left(\eta_{\mathrm{eff}}\right)$, which is the power-law index that relates the driving continuum flux to the responding emissionline fluxes,

$$
\log F_{\text {line }}=A+\eta_{\text {eff }}\left[\log F_{\text {cont }}\right] .
$$

In the case of no line response, $\eta_{\text {eff }}=0$, and if the line responds linearly to continuum variations (i.e., the transfer function is a $\delta$ function), then $\eta_{\mathrm{eff}}=1$. The emission-line EW and the continuum flux are related via the Baldwin relation 
(Baldwin 1977), which is described by

$$
\log \mathrm{EW}_{\text {line }}=B+\beta\left[\log F_{\text {cont }}\right],
$$

where the choice for $F_{\text {cont }}$ is assumed to be a reasonable proxy for the ionizing continuum. Thus, $\beta$ is also known as the slope of the Baldwin relation.

Following the same procedures as in Paper IV, we compute the responsivity $\eta_{\mathrm{eff}}$ and $\mathrm{EW}$ for the portion of the $\mathrm{H} \beta$ light curve that correlates with the UV continuum and then examine how these values change in different segments of the light curves. The values $F_{\text {cont }}$ and $F_{\text {line }}$ refer to the continuum and emission-line fluxes after removing nonvariable components such as host-galaxy and narrow-line flux contributions, and after correcting for the mean time delay between the continuum and line light curves (Pogge \& Peterson 1992; Gilbert \& Peterson 2003; Goad et al. 2004). There is very little hostgalaxy flux in the $1158 \AA$ continuum, which is dominated by the variable AGN. For the line fluxes, we took $\mathrm{H} \beta$ fluxes measured after linear continuum removal (without spectral decomposition) and subtracted a constant narrow $\mathrm{H} \beta$ flux measured from the MDM mean spectrum fit to remove the nonvariable line component. To correct for the emission-line time delay, we shifted the $\mathrm{H} \beta$ light curve by 8 days, which corresponds to the lag for the portion of the line light curve closely correlated with the UV continuum. Figure 12(a) shows the $1158 \AA$ continuum light curve (black) with the time-shifted and flux-scaled $\mathrm{H} \beta$ light curve, which has been truncated at the beginning to match the first epoch of continuum observations. To show the $\mathrm{H} \beta$ light curve's general behavior toward the end of the campaign, we have shown here the full 143 epochs of $\mathrm{H} \beta$ flux measurements instead of the 133-epoch light curve we used in the $\mathrm{H} \beta$ lag analysis. However, since the last 10 epochs of spectra suffer from inconsistent spectral flux calibration (see Section 2), we do not use these points in calculating $\eta_{\text {eff }}$ or $\beta$.

We divided the $\mathrm{H} \beta$ light curve into five segments. The first segment corresponds to the period when the line light curve closely follows the continuum light curve (blue points); the second and third segments correspond to periods when the line light curve decouples from the continuum (cyan points) and remains in a state of depressed flux (red points); the last two segments correspond to the line light curve recovering from the depressed state (magenta points) and correlating once again with the continuum light curve (green points). The epochs that divide these segments are THJD $=[6743,6772,6812,6827]$.

Figures 12(d) and (e) show the $\mathrm{H} \beta$ broad-line flux and EW as a function of the $1158 \AA$ continuum flux density determined from the HST epoch closest to each MDM epoch. The red lines represent linear least-squares fits to Equations (8) and (9) using only the blue points. We found that the cyan, red, and magenta points-corresponding to when the light curves are not well correlated-lie well below the best fits of Equations (8) and (9) to the blue points. Furthermore, the epochs during the anomaly (red points) are characterized by an $\eta_{\text {eff }}$ value consistent with zero $\left(\eta_{\text {eff }}=0.02 \pm 0.03\right.$; black dotted line in Figure 12(d)), which shows that the emission-line strength remained constant independent of continuum strength. Similar results were found for the $\mathrm{C}$ IV, $\mathrm{Ly} \alpha$, He II $(+\mathrm{O} I I I])$, and $\mathrm{Si} \mathrm{IV(+O} \mathrm{IV])} \mathrm{emission}$ lines in Paper IV. Table 6 summarizes the $\eta_{\mathrm{eff}}$ and $\beta$ values for all broad emission lines measured during this campaign. Note that the values from Paper IV were computed using epochs both before and after the anomaly (blue and green points),
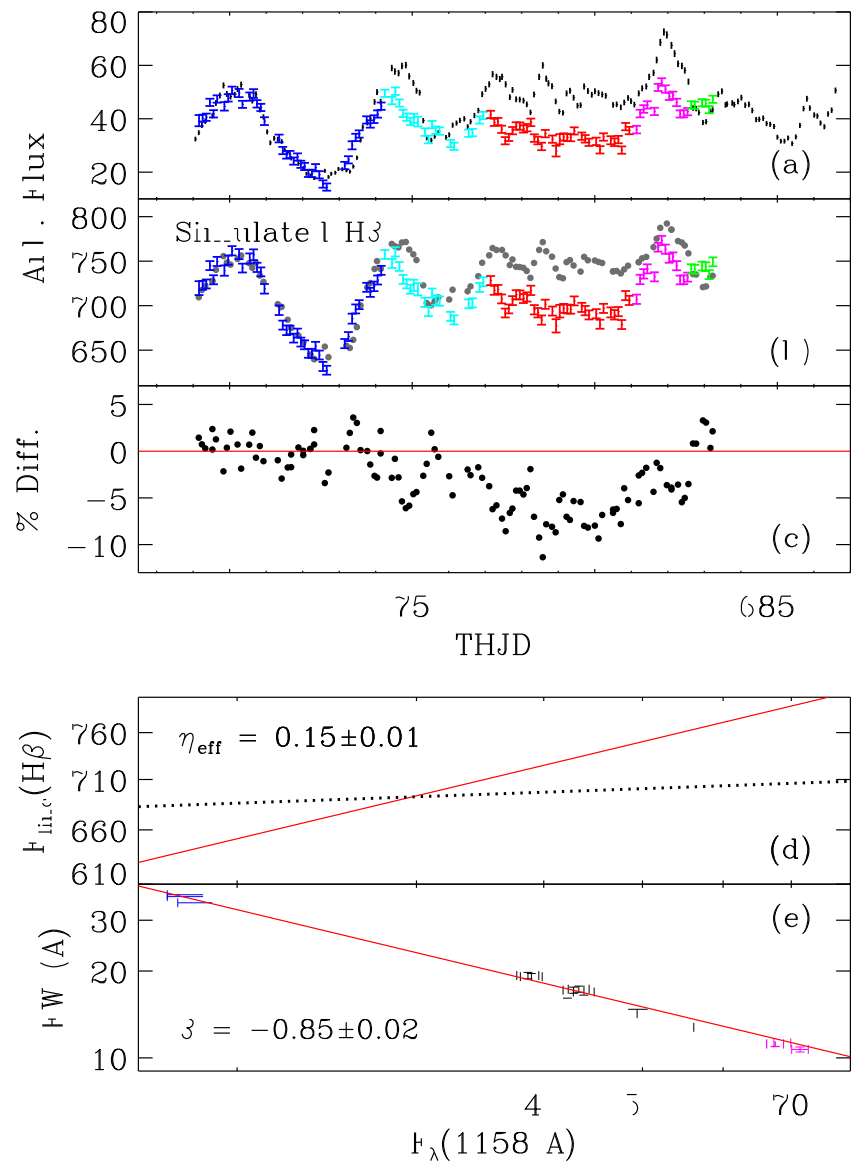

Figure 12. (a) $1158 \AA$ continuum light curve with the time-shifted $\mathrm{H} \beta$ light curve (see text for color scheme). (b, c) Reconstructed $\mathrm{H} \beta$ light curve (gray dots) and the percent of flux lost during the anomaly, respectively. (d, e) $\mathrm{H} \beta$ broad-line flux $\left(10^{-15} \mathrm{erg} \mathrm{s}^{-1} \mathrm{~cm}^{-2}\right)$ and EW $(\AA)$ as a function of the $1158 \AA$ continuum flux density $\left(10^{-15} \mathrm{erg} \mathrm{s}^{-1} \mathrm{~cm}^{-2} \AA^{-1}\right)$. The solid red lines are linear least-squares fits to the blue points, which are from the period when the line and continuum light curves are well correlated. The slopes of these fits are shown in each panel. The dotted black line in panel (d) is the least-squares fit to the red points (anomaly) and has a slope of $\eta_{\text {eff }}=0.02 \pm 0.03$.

Table 6

Responsivity, Slope of the Baldwin Relation, and Flux Deficit during the Anomaly for All Emission Lines

\begin{tabular}{lccr}
\hline \hline Line ID & $\eta_{\text {eff }}$ & $\beta$ & $F_{\text {lost }}$ \\
\hline Ly $\alpha$ & $0.30 \pm 0.01$ & $-0.73 \pm 0.02$ & $9 \%$ \\
Si IV+O IV] & $0.45 \pm 0.01$ & $-0.58 \pm 0.03$ & $23 \%$ \\
C IV & $0.25 \pm 0.01$ & $-0.75 \pm 0.01$ & $18 \%$ \\
He II+O III] & $0.58 \pm 0.04$ & $-0.48 \pm 0.04$ & $21 \%$ \\
H $\beta$ & $0.15 \pm 0.01$ & $-0.85 \pm 0.02$ & $6 \%$ \\
\hline
\end{tabular}

Note. All values were measured using the $1158 \AA$ continuum fluxes from this campaign. The first four rows show values from Paper IV.

whereas our values were calculated using only epochs before the anomaly (blue points).

We measured the amplitude of the anomaly by comparing the observed $\mathrm{H} \beta$ light curve with a simulated light curve that represents what the line response would have been without the anomaly. The simulated line fluxes $\left(F_{\text {sim }}\right)$ were calculated using the observed continuum fluxes and Equation (8), where $\eta_{\text {eff }}$ and $A$ are chosen to be the best-fit values for the period 
Table 7

$\mathrm{H} \beta$ Responsivity and AGN Optical Continuum Flux Density for NGC 5548 over $25 \mathrm{yr}$

\begin{tabular}{cccc}
\hline \hline Year & $\eta_{\text {eff }}$ & Mean $F_{\text {cont }}$ & rms $F_{\text {cont }}$ \\
\hline 1989 & $0.56 \pm 0.04$ & 6.54 & 1.27 \\
1990 & $0.84 \pm 0.03$ & 3.79 & 0.91 \\
1991 & $0.95 \pm 0.09$ & 6.06 & 0.92 \\
1992 & $0.94 \pm 0.05$ & 3.34 & 1.17 \\
1993 & $0.43 \pm 0.04$ & 5.69 & 0.87 \\
1994 & $0.74 \pm 0.04$ & 6.40 & 1.11 \\
1995 & $0.68 \pm 0.04$ & 8.71 & 1.01 \\
1996 & $0.54 \pm 0.03$ & 7.07 & 1.52 \\
1997 & $0.80 \pm 0.07$ & 4.73 & 0.89 \\
1998 & $0.51 \pm 0.02$ & 10.05 & 1.44 \\
1999 & $0.41 \pm 0.04$ & 8.48 & 1.82 \\
2000 & $0.65 \pm 0.06$ & 3.59 & 1.20 \\
2001 & $1.00 \pm 0.12$ & 3.65 & 0.86 \\
2014 & $0.59 \pm 0.01$ & 7.44 & 0.50 \\
\hline
\end{tabular}

Note. Flux densities are in units of $10^{-15} \mathrm{erg} \mathrm{s}^{-1} \mathrm{~cm}^{-2} \AA^{-1}$. The first 13 rows show values from Goad et al. (2004), and the last row shows values from this campaign. All $\eta_{\text {eff }}$ values listed were measured with respect to the $5100 \AA$ continuum.

when the light curves are well correlated (blue points). This simulated light curve is shown in Figure 12(b) with gray dots, and comparing it with the observed data (colored points) shows that the $\mathrm{H} \beta$ line had lower-than-expected variability amplitude and mean flux during T2. If we define the fractional flux loss as $F_{\text {lost }}=\left(F_{\text {sim }}-F_{\text {obs }}\right) / F_{\text {sim }}$ (Figure $\left.12(\mathrm{c})\right)$, then this implies an $\mathrm{H} \beta$ flux deficit of $\sim 6 \%$ during the anomaly (red points), which is close to the deficit for $\operatorname{Ly} \alpha$ but much smaller than that of the other UV emission lines, as shown in Table 6.

Table 7 summarizes the $\mathrm{H} \beta$ responsivities and AGN optical continuum flux densities measured by Goad et al. (2004) for every year of the $13 \mathrm{yr}$ monitoring campaign carried out by the AGN Watch consortium (Peterson et al. 2002), ${ }^{95}$ along with the values of $\eta_{\mathrm{eff}}=0.59 \pm 0.01$ and $\left\langle F_{5100, \mathrm{AGN}}\right\rangle=(7.44 \pm 0.50) \times$ $10^{-15} \mathrm{erg} \mathrm{s}^{-1} \mathrm{~cm}^{-2} \AA^{-1}$ calculated from this data set. Both the responsivity and optical continuum flux density for this campaign are close to those measured from the 1998 data. Using the mean $\mathrm{H} \beta$ flux without narrow-line contributions of $\left\langle F_{\mathrm{H} \beta}\right\rangle=(690 \pm 2.40) \times 10^{-15} \mathrm{erg} \mathrm{s}^{-1} \mathrm{~cm}^{-2}$, we find a mean EW for this campaign of $92.75 \pm 2.45 \AA$, which is lower than values measured by Goad \& Korista (2014) for previous campaigns.

The $\mathrm{H} \beta$ light curve appears to decorrelate from the UV continuum light curve at a somewhat earlier time $(\mathrm{THJD} \approx 6742)$ than $\mathrm{C}$ IV $($ THJD $\approx 6765$ as found in Paper IV). If we assume that the $\mathrm{H} \beta$ light curve decorrelates and recorrelates with the continuum light curve at the same times as C IV and use the same dividing epochs as those used in Paper IV $\quad$ (THJD $=[6766,6777,6814,6830])$, then $\eta_{\text {eff }}=0.13 \pm 0.01$ and $\beta=-0.88 \pm 0.01$ for the blue points.

While He II $\lambda 4686$ also shows anomalous behavior during the T2 period (Figure 7), its broad-line component is very weak and the fitted profile is poorly constrained in the spectral decomposition process. The He II light curve is thus noisier than that of $\mathrm{H} \beta$, and the $F_{\text {line }}$ and $\mathrm{EW}$ values are poorly

\footnotetext{
95 The continuum flux densities from Peterson et al. (2002) have been updated by Bentz et al. (2013) and Kilerci Eser et al. (2015).
}

correlated with $F_{\text {cont }}$. We therefore do not perform a detailed analysis of the responsivity for He II.

\subsection{Line Width and $M_{B H}$ Estimate}

The BH mass in NGC 5548 has been estimated for several previous RM campaigns, including the AGN Watch consortium (Peterson et al. 2002), Bentz et al. (2007, 2009b), and Denney et al. (2010). The AGN Watch group determined the BH mass using data from each of the program's monitoring years, and subsequent campaigns each produced an independent $\mathrm{BH}$ mass value. Here we compute the $\mathrm{BH}$ mass using data from this campaign and compare it with previous results.

We measured the line widths from the $\mathrm{H} \beta$ mean and rms spectra as shown in Figure 5. The narrow $\mathrm{H} \beta$ line essentially disappears in the rms spectrum but is still present in the mean spectrum. We removed this emission component by subtracting a Gaussian fit to the $\mathrm{H} \beta$ narrow line in the mean MDM spectrum, and then linearly interpolated over the narrow $\mathrm{H} \beta$ residuals and the [O III] $\lambda \lambda 4959$ and 5007 residuals.

Two emission-line width values are typically measured in RM: the FWHM and the line dispersion, defined by

$$
\sigma_{\text {line }}^{2}=\left(\frac{c}{\lambda_{0}}\right)^{2}\left(\frac{\sum \lambda_{i}^{2} S_{i}}{\sum S_{i}}-\lambda_{0}^{2}\right)
$$

where $S_{i}$ is the flux density at wavelength bin $\lambda_{i}$ and $\lambda_{0}$ is the flux-weighted centroid wavelength of the line profile. We measured $\sigma_{\text {line }}$ and FWHM for the mean and rms spectra using the line profile within the rest-frame wavelength range 4669.8-5063.0 $\AA$. We treated the mean profile as double peaked and followed the procedures described by Peterson et al. (2004) to measure its FWHM. From each of the two peaks at 4827.1 and $4886.1 \AA$, we traced the line profile outward until the flux reached $0.5 F_{\max }$, and then traced the line profile inward from the continuum until the flux again reached $0.5 F_{\text {max }}$. The two wavelengths at $0.5 F_{\max }$ - which generally agree well for a smooth line profile-are then averaged to obtain the wavelength at half-maximum on each side of the profile. The rms profile is more complicated because it has more than two peaks and the troughs between them can reach well below half of the peak fluxes. We therefore identified a single maximum flux $F_{\text {max }}$ and traced the profile from the continuum toward the center on both sides until the flux reached $0.5 F_{\max }$. The separation between the two wavelengths at $0.5 F_{\max }$ is taken to be the FWHM of the rms spectrum.

The $\mathrm{H} \beta$ line widths and their uncertainties were determined using Monte Carlo bootstrap analysis. With $n$ total spectra, we randomly selected $n$ spectra from the data set with replacement, constructed mean and rms line profiles, and measured the line dispersion and FWHM. The median and standard deviation of $10^{4}$ bootstrap realizations are used for the $\sigma_{\text {line }}$ and FWHM and their estimated uncertainties.

There are additional systematic uncertainties in the line widths from using different Fe II templates in the spectral decomposition. We repeated the bootstrap analysis after performing spectral decompositions using each of the Boroson \& Green (1992), Véron-Cetty et al. (2004), and Kovačević et al. (2010) Fe II templates, and then took the standard deviation of the $\mathrm{H} \beta$ widths from using the different templates as the systematic uncertainty for the line width. This systematic error dominates the error budget for all $\sigma_{\text {line }}$ measurements and 
Table 8

$\mathrm{H} \beta$ Rest-frame Line Widths

\begin{tabular}{llcc}
\hline \hline Segment & Spectrum & $\begin{array}{c}\sigma_{\text {line }} \\
\left(\mathrm{km} \mathrm{s}^{-1}\right)\end{array}$ & $\begin{array}{c}\text { FWHM } \\
\left(\mathrm{km} \mathrm{s}^{-1}\right)\end{array}$ \\
\hline Full & rms & $4278 \pm 671$ & $10161 \pm 587$ \\
T1 & rms & $4155 \pm 513$ & $10861 \pm 739$ \\
T2 & rms & $4856 \pm 731$ & $9103 \pm 1279$ \\
Full & Mean & $3691 \pm 162$ & $9496 \pm 418$ \\
T1 & Mean & $3983 \pm 150$ & $9612 \pm 427$ \\
T2 & Mean & $3939 \pm 177$ & $9380 \pm 158$ \\
\hline
\end{tabular}

the FWHM of the mean spectrum, but is comparable to the uncertainty from bootstrapping analysis for the FWHM of the rms spectrum. We added this systematic uncertainty in quadrature to the statistical uncertainty from the Kovačević et al. (2010) line width to obtain the final $\mathrm{H} \beta$ line width uncertainty.

The line widths are also affected by instrumental broadening due to the use of a wide slit. The observed line width is the quadratic sum of the intrinsic and instrumental line widths $\left(\sigma_{\text {observed }}^{2}=\sigma_{\text {intrinsic }}^{2}+\sigma_{\text {instrumental }}^{2}\right.$ or similarly for FWHM). To calculate the instrumental broadening for a $5^{\prime \prime}$ slit, we followed the methods described by Bentz et al. (2009b) and compare the [O III] $\lambda 5007$ line width measured from our observations with the width measured from a higher-resolution observation taken using a narrow $\left(\sim 2^{\prime \prime}\right)$ slit, which represents the intrinsic line width. The FWHM of the [O III] $\lambda 5007$ model from the MDM rest-frame mean spectrum is $9.79 \AA\left(572 \mathrm{~km} \mathrm{~s}^{-1}\right)$, while Whittle (1992) found an FWHM of $410 \mathrm{~km} \mathrm{~s}^{-1}$ using a $2^{\prime \prime}$ slit. This implies an instrumental broadening of $\mathrm{FWHM}_{\text {instrument }}=399 \mathrm{~km} \mathrm{~s}^{-1}$, corresponding to $\sigma_{\text {instrument }}=170 \mathrm{~km} \mathrm{~s}^{-1}$ for a Gaussian model of the [O III] line profile. We subtract this instrumental width in quadrature from the $\mathrm{H} \beta$ line width measurements to obtain the intrinsic $\mathrm{H} \beta$ line widths, which are listed in Table 8. The large uncertainties on the FWHM measurements from the rms spectrum are a result of the jagged shape of the rms profile.

We use the $\mathrm{H} \beta$ line dispersion measured from the rms spectrum as the velocity dispersion $\Delta V$, as is common practice in RM (Peterson et al. 2004; but also see Collin et al. 2006; Mejía-Restrepo et al. 2016, for comparison of FWHM and sigma as indicators of BLR virial velocity for $\mathrm{BH}$ mass estimation), and calculate the virial product, defined as $\mathrm{VP}=c \tau \Delta V^{2} / G$. The $f$ factor in Equation (2), which incorporates the geometry and kinematics of the BLR, is generally unknown for any individual AGN, so a single value $\langle f\rangle$ is often used to represent the average normalization for all AGNs. This value is usually taken to be the scale factor that puts the sample of RM virial products onto the same $M_{\mathrm{BH}}-\sigma_{\star}$ relation as nearby inactive galaxies (see Kormendy \& Ho 2013, for a discussion of the uncertainties in the $M_{\mathrm{BH}}-\sigma_{\star}$ relation), and can vary depending on the sample of AGNs used in the fit, as well as the fitting method (Onken et al. 2004; Watson et al. 2007; Woo et al. 2010; Graham et al. 2011; Park et al. 2012a; Grier et al. 2013; Ho \& Kim 2015). We adopt a value of $\langle f\rangle=4.47$ as calculated by Woo et al. (2015). Since $\langle f\rangle$ is calibrated using $\mathrm{H} \beta$ lags measured against the optical continuum, we used the $\mathrm{H} \beta-5100 \AA$ lag to calculate the VP and $\mathrm{BH}$ mass.

Table 9 lists the virial products and the inferred $\mathrm{BH}$ masses for the full, T1, and T2 segments. Our $M_{\mathrm{BH}}$ uncertainties do not include uncertainties in $\langle f\rangle$ (Woo et al. 2015) or scatter in the distribution of $f$ for different AGNs (e.g., Ho \& Kim 2015). The $\mathrm{T} 1$ and $\mathrm{T} 2 M_{\mathrm{BH}}$ estimates are consistent even though the two lags are different by more than $1 \sigma$. Compared to recent measurements, the full-campaign virial product $\left(1.49_{-0.49}^{+0.49} \times 10^{7} M_{\odot}\right)$ is entirely consistent with the values of $1.50_{-0.51}^{+0.37} \times 10^{7} M_{\odot}$ and $1.38_{-0.41}^{+0.51} \times 10^{7} M_{\odot}$ obtained by Bentz et al. (2009b) and Lu et al. (2016), respectively. Our BH mass measurement $\left(6.66_{-2.17}^{+2.17} \times 10^{7} M_{\odot}\right)$ is also consistent to $1 \sigma$ with the dynamical mass of $3.24_{-0.90}^{+2.26} \times 10^{7} M_{\odot}$ as determined by Pancoast et al. (2014), who use the $V$-band continuum as the ionizing source but do not use the $f$ factor, which compensates for the difference in lag between using the UV and optical continua (see next section). This will lead to an underestimate of $R_{\mathrm{BLR}}$ and hence a proportional underestimate of the $\mathrm{BH}$ mass (Equation (2)). If we scale the Pancoast et al. (2014) mass by the ratio between the $\mathrm{H} \beta-1158 \AA$ and the $\mathrm{H} \beta-V$-band lags from Table $4\left(\tau_{\mathrm{H} \beta-\mathrm{UV}} /\right.$ $\tau_{\mathrm{H} \beta-V}=1.64 \pm 0.12$ ), then the two $M_{\mathrm{BH}}$ measurements become much more congruent.

\section{Discussion}

\subsection{Implications of $U V$ and Optical $H \beta$ Lags}

Ground-based RM campaigns have traditionally used the optical continuum light curve-by necessity-to determine emission-line lags, even though the far-UV continuum is a better proxy for the ionizing source. Lags relative to the UV continuum $\left(\tau_{\mathrm{H} \beta-\mathrm{UV}}\right)$ thus should yield more accurate estimates of the BLR characteristic radius than lags measured relative to the optical continuum. Our $\mathrm{H} \beta-\mathrm{UV}$ lag $\left(\tau_{\mathrm{H} \beta-\mathrm{UV}}=6.23_{-0.44}^{+0.39}\right.$ days $)$ is $\sim 2$ days longer than the $\mathrm{H} \beta$-optical lag $\left(\tau_{\mathrm{H} \beta-\text { opt }}=4.17_{-0.36}^{+0.36}\right.$ days). Given that past measurements of the $\mathrm{H} \beta$-optical lag for this object range from $\sim 4$ to $\sim 25$ days (Bentz et al. 2013, and references therein), and assuming that $\tau_{\mathrm{H} \beta \text {-opt }}$ is always $\sim 2$ days longer than $\tau_{\mathrm{H} \beta-\mathrm{UV}}$, the BLR characteristic radius estimated from previous campaigns using only optical data is biased low by $10 \%-50 \%$. The difference in these $\mathrm{H} \beta$ lags is also consistent with the optical-to-UV continuum lag of $2.24_{-0.24}^{+0.24}$ days found in Paper III.

Since virial estimates of $M_{\mathrm{BH}}$ scale with $R_{\mathrm{BLR}}$, it may seem that this difference between $\tau_{\mathrm{H} \beta-\mathrm{UV}}$ and $\tau_{\mathrm{H} \beta-\mathrm{opt}}$ will change the $\mathrm{BH}$ mass estimate for NGC 5548 and other reverberationmapped AGNs. However, the virial product—not the BH mass - is the quantity that is directly affected, and the normalization factor $f$ is still needed to scale the virial product to a calibrated $\mathrm{BH}$ mass (Equation (2)). If the ratio of $\tau_{\mathrm{H} \beta-\mathrm{UV}} / \tau_{\mathrm{H} \beta \text {-opt }}$ is the same for all AGNs, then all RM virial products would be scaled up by a constant value, so simply changing the value of $f$ would remove this bias and leave the RM BH masses unchanged. Even if the lag ratio is not constant for all AGNs, its effect on the $\mathrm{BH}$ mass scale is still small because the largest source of $M_{\mathrm{BH}}$ uncertainty in $\mathrm{RM}$ is the calibration uncertainty for $f$ at $\sim 0.12$ dex (Woo et al. 2015). Furthermore, for NGC 5548, the $\mathrm{H} \beta$ lag measured during this campaign is relatively short compared to its historical values (see Section 5.3). If the lag had been longer, the ratio of $\tau_{\mathrm{H} \beta-\mathrm{UV}} / \tau_{\mathrm{H} \beta-\text { opt }}$ would be closer to unity and the bias in the BLR characteristic size and BH mass estimate would be much smaller.

While the discrepancy between $\tau_{\mathrm{H} \beta-\mathrm{UV}}$ and $\tau_{\mathrm{H} \beta-\mathrm{opt}}$ may not change $M_{\mathrm{BH}}$ measurements that use the $f$ factor, dynamical models that directly infer $M_{\mathrm{BH}}$ and BLR characteristics (e.g., Pancoast et al. 2011; Li et al. 2013) using only optical continuum data are affected since they do not depend on this 
Table 9

$\mathrm{H} \beta$ Line Measurements, $M_{\mathrm{BH}}$ Estimates, and Continuum Flux Densities

\begin{tabular}{|c|c|c|c|c|c|c|}
\hline Segment & $\begin{array}{c}\sigma_{\text {line }} \\
\left(\mathrm{km} \mathrm{s}^{-1}\right)\end{array}$ & $\begin{array}{r}\tau_{\mathrm{H} \beta \text {-opt }} \\
\text { (days) }\end{array}$ & $\begin{array}{r}\text { Virial Product } \\
\quad\left(10^{7} M_{\odot}\right)\end{array}$ & $\begin{array}{r}M_{\mathrm{BH}} \\
\left(10^{7} M_{\odot}\right)\end{array}$ & \multicolumn{2}{|c|}{$\begin{array}{l}F_{5100, \text { total }} \\
\quad\left(10^{-15} \mathrm{erg} \mathrm{s}^{-1} \mathrm{~cm}^{-2} \AA^{F_{5100, \mathrm{AGN}}}{ }^{-1}\right)\end{array}$} \\
\hline Full & $4278 \pm 671$ & $4.17_{-0.36}^{+0.36}$ & $1.49_{-0.49}^{+0.49}$ & $6.66_{-2.17}^{+2.17}$ & $11.96 \pm 0.07$ & $7.44 \pm 0.50$ \\
\hline $\mathrm{T} 1$ & $4155 \pm 513$ & $4.99_{-0.47}^{+0.40}$ & $1.68_{-0.45}^{+0.44}$ & $7.53_{-1.99}^{+1.96}$ & $11.31 \pm 0.08$ & $6.79 \pm 0.46$ \\
\hline $\mathrm{T} 2$ & $4856 \pm 731$ & $3.10_{-0.80}^{+0.77}$ & $1.43_{-0.56}^{+0.55}$ & $6.38_{-2.53}^{+2.49}$ & $12.51 \pm 0.04$ & $7.99 \pm 0.45$ \\
\hline
\end{tabular}

Note. $\tau_{\mathrm{H} \beta \text {-opt }}$ is the rest-frame ICCF $\tau_{\text {cen }}$ value measured against the $5100 \AA$ continuum.

virial normalization. The BLR characteristic size for each AGN inferred using optical data alone would thus be biased by a factor that depends on the value of $\tau_{\mathrm{H} \beta-\mathrm{UV}} / \tau_{\mathrm{H} \beta-\mathrm{opt}}$ for that particular object. Changes in the inferred $R_{\mathrm{BLR}}$ could also impact single-epoch $M_{\mathrm{BH}}$ estimates, which rely on the empirical relation between the BLR characteristic radius and the AGN continuum luminosity $\left(R_{\mathrm{BLR}} \propto L_{\mathrm{AGN}}^{\alpha}\right.$ with $\alpha \approx 1 / 2$; e.g., Laor 1998; Wandel et al. 1999; Kaspi et al. 2000, 2005; McLure \& Jarvis 2002; Bentz et al. 2006, 2009a, 2013; Vestergaard \& Peterson 2006). If $\tau_{\mathrm{H} \beta-\mathrm{UV}} / \tau_{\mathrm{H} \beta-\mathrm{opt}}$ correlates with AGN luminosity, then the expected value of $\alpha$ would change, and if this ratio is different for all AGNs but is uncorrelated with any other AGN properties, then this would introduce additional scatter to the scaling relation.

We can examine the expected scaling of $\tau_{\mathrm{H} \beta-\mathrm{UV}} / \tau_{\mathrm{H} \beta-\mathrm{opt}}$ with respect to $M_{\mathrm{BH}}$ and $L_{\mathrm{AGN}}$ by using simple disk and BLR ionization models. Assuming that $\tau_{\mathrm{H} \beta-\mathrm{UV}}$ is the sum of $\tau_{\mathrm{H} \beta-\mathrm{opt}}$ and the optical-UV interband continuum lag $\tau_{\mathrm{opt}-\mathrm{UV}}$, then

$$
\frac{\tau_{\mathrm{H} \beta-\mathrm{opt}}}{\tau_{\mathrm{H} \beta-\mathrm{UV}}}=1-\frac{\tau_{\mathrm{opt}-\mathrm{UV}}}{\tau_{\mathrm{H} \beta-\mathrm{UV}}}
$$

For a standard thin disk, the characteristic size scale of the disk region emitting at wavelength $\lambda$ scales as

$$
R_{\lambda} \propto M_{\mathrm{BH}}^{2 / 3} l^{1 / 3} \lambda^{4 / 3},
$$

where $l=L / L_{\text {Edd }}$. For a simple photoionization equilibrium model, the BLR characteristic radius scales as

$$
R_{\mathrm{BLR}} \propto M_{\mathrm{BH}}^{1 / 2} l^{1 / 2} .
$$

If the interband continuum lags are due to light-travel time across the accretion disk, then

$$
\frac{\tau_{\mathrm{opt}-\mathrm{UV}}}{\tau_{\mathrm{H} \beta-\mathrm{UV}}} \propto M_{\mathrm{BH}}^{1 / 6} l^{-1 / 6} \lambda^{4 / 3}
$$

The lag ratio is thus weakly dependent on both $M_{\mathrm{BH}}$ and accretion rate, where even a factor of $10^{3}$ increase in the $\mathrm{BH}$ mass or accretion rate will change the ratio by only a factor of three. Empirically, Bentz et al. (2013) found a low scatter of 0.13 dex around the $R_{\mathrm{BLR}}-L_{\mathrm{AGN}}$ relation for $41 \mathrm{AGNs}$ over four orders of magnitude in luminosity, which suggests that this effect is indeed small for most AGNs. However, it is important to directly examine the potential consequences of this effect by obtaining more simultaneous observations of the UV/optical continua and the broad emission lines for AGNs over a wide range of luminosities.

\subsection{Anomalous Emission-line Light-curve Behavior}

Despite apparent differences in the $\mathrm{C}$ IV and $\mathrm{H} \beta$ decorrelation start times and flux deficits during T2, it is likely that the cause of the anomalous light-curve behavior is the same for the UV and optical emission lines. The line response during the anomaly is also heavily dependent on the line-of-sight velocity for $\mathrm{H} \beta$ (Figure 10) and the UV emission lines (M. Goad et al. 2017 , in preparation). Paper IV suggests two scenarios that could produce the anomaly: (1) a temporary obscuration of the ionizing source from parts of the BLR by a moving veil of gas between the accretion disk and BLR, or (2) a temporary change in spectral energy distribution of the ionizing source. Future papers will investigate in detail the timing and magnitude of the anomaly for all UV and optical lines using the full multiwavelength data set from this campaign.

The bottom panel of Figure 7 shows that the $\mathrm{H} \beta$ light-curve decorrelation was also clearly detected using only the optical data. If our campaign had lasted for only the duration of T2, we still would have been able to measure the $\mathrm{H} \beta$ lags with fairly high precision $\left(\tau_{\mathrm{H} \beta-\mathrm{UV}}=5.99_{-0.75}^{+0.71}\right.$ days and $\tau_{\mathrm{H} \beta-\mathrm{opt}}=$ $3.10_{-0.80}^{+0.77}$ days), but the lag signal would be contaminated by other unknown factors and would lead to a somewhat biased estimate of the BLR characteristic radius. Depending on how common this decorrelation behavior is for $\mathrm{H} \beta$, this effect could contribute to additional scatter in the single-object $R_{\mathrm{BLR}}-L_{\mathrm{AGN}}$ relations for NGC 5548 and other AGNs, which can account for about half of the observed scatter in the global $R_{\mathrm{BLR}}-L_{\mathrm{AGN}}$ relation for the entire sample of reverberation-mapped AGNs (Kilerci Eser et al. 2015).

The high cadence and long duration of this campaign have both been crucial in detecting this decorrelation phenomenon. Horne et al. (2004) found that a campaign duration of at least three times the maximum BLR light-crossing time is needed to recover high-fidelity velocity-delay maps from reverberation mapping data. Given the $\mathrm{H} \beta-\mathrm{UV}$ lag of $\sim 6$ days for NGC 5548 during our monitoring period, the BLR characteristic lightcrossing time is $\sim 12$ days, and the minimum campaign length needed to recover velocity-delay maps would correspond to $\sim 40$ days, which would not have allowed us to see this decorrelation. In order to detect and characterize these anomalous behaviors in the emission-line light curves, RM campaigns must be much longer than the minimum requirement for obtaining velocity-delay maps.

Finally, while there are no previously published results documenting similar emission-line light-curve behavior, it is possible that this decorrelation phenomenon was indeed observed in other AGNs in previous RM campaigns, but was not recognized as such because the campaign had relatively low cadence and/or short duration. RM programs designed to study large numbers of sources with lower cadence would also 


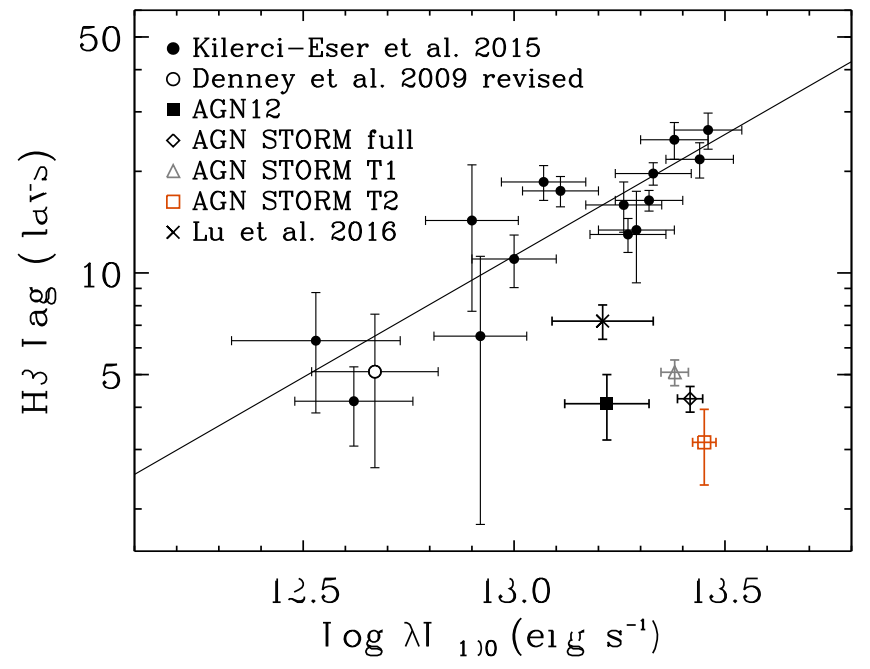

Figure 13. NGC 5548 optical continuum luminosity and $\mathrm{H} \beta$-optical lags from all monitoring campaigns to date. The solid line is the linear least-squares fit to the Kilerci Eser et al. (2015) and Denney et al. (2009) points.

not be able to detect these decorrelations. This further highlights the importance of high-cadence, long-duration, and high-S/N multiwavelength reverberation data sets in order to determine the prevalence of this phenomenon.

\subsection{Comparison to Previous Campaigns: The $R_{B L R}-L_{A G N}$ Relation}

We compare the NGC $5548 \mathrm{H} \beta$-optical lags and optical continuum luminosity $\left(L_{5100}\right)$ from this campaign with those from previous RM campaigns targeting this object, as compiled by Kilerci Eser et al. (2015). The total $5100 \AA$ flux and the AGN continuum flux densities for the full, T1, and T2 segments are listed in the sixth and seventh columns of Table 9, respectively. We applied a Galactic extinction correction of $E$ $(B-V)=0.017 \mathrm{mag}$ (Schlegel et al. 1998; Schlafly \& Finkbeiner 2011) and used a luminosity distance of $75 \mathrm{Mpc}$ in converting fluxes to luminosities. Figure 13 shows the $R_{\mathrm{BLR}}{ }^{-}$ $L_{\mathrm{AGN}}$ relation for NGC 5548, where the uncertainties are from absolute photometric calibration using [O III] $\lambda 5007$ and do not include the luminosity distance uncertainty. The lags from this campaign have smaller uncertainties, due to the high cadence and long duration of the ground-based monitoring. Denney et al. (2010) monitored NGC 5548 as part of a multiobject RM campaign and found $\tau_{\mathrm{H} \beta-\text { opt }}=12.40_{-3.85}^{+2.74}$ days. However, the light curves were dominated by a large, long-term trend, and after detrending the light curves with a third-order polynomial, the $\mathrm{H} \beta$ lag becomes $5.07_{-2.37}^{+2.46}$ days (open circle in Figure 13).

The surprising result is that, given the AGN luminosity during this campaign, the $\mathrm{H} \beta$ lags are nearly five times shorter than expected based on past measurements. NGC 5548 was also monitored in 2012 (G. De Rosa et al. 2017, in preparation) and had an $\mathrm{H} \beta$ lag of $4.1 \pm 0.9$ days and a luminosity of $\log (\lambda$ $\left.L_{5100}\right)=43.22 \pm 0.1$, whereas Lu et al. (2016) found the $\mathrm{H} \beta$ lag to be $7.2_{-0.35}^{+1.33}$ days and the AGN luminosity to be $\log (\lambda$ $\left.L_{5100}\right)=43.21 \pm 0.1$ during their 2015 campaign, as shown in Figure 13. Both of these lags are also shorter than expected based on past results, though the point from Lu et al. (2016) suggests that the AGN may be returning to its previously measured $R_{\mathrm{BLR}}-L_{\mathrm{AGN}}$ relation.

In numerical simulations of the emission-line response to continuum variations for a model BLR with fixed radial extent,
Goad \& Korista (2014) found that if the characteristic continuum variability timescale $\left(\tau_{\text {char }}\right)$ is smaller than the BLR light-crossing time, then there exists a strong correlation between $\tau_{\text {char }}$, the line responsivity $\left(\eta_{\text {eff }}\right)$, and measured lag, such that both $\eta_{\text {eff }}$ and the lag decrease as $\tau_{\text {char }}$ decreases (their Figure 9). This is because short-timescale continuum variations only probe the inner parts of the BLR, so the measured lag and responsivity would be biased low. If we use the FWHM of the continuum light-curve autocorrelation function as a crude proxy for the characteristic variability timescale, then $\tau_{\text {char }} \sim 10$ days, which is significantly shorter than the values measured for this source for the 1989 IUE campaign and the 1993 HST campaign and could explain the shorter-thanexpected $\mathrm{H} \beta$ lags.

Regardless of the physical causes of the short lags, these results suggest that the $R_{\mathrm{BLR}}-L_{\mathrm{AGN}}$ relation is more complex than previously realized. Bentz et al. (2013) found a tight correlation between $R_{\mathrm{BLR}}$ and $L_{\mathrm{AGN}}$ for a sample of $\sim 40$ reverberation-mapped AGNs, but recent studies by Du et al. $(2015,2016 b)$ have shown that many AGNs with very high accretion rates tend to have considerably shorter $\mathrm{H} \beta$ lags compared to low-accretion AGNs with similar luminosities. Now, we have shown that even for a single AGN with low accretion rate $\left(\lambda_{\mathrm{Edd}}=0.021\right.$ for $\mathrm{NGC} 5548$; Vasudevan et al. 2010), the $R_{\mathrm{BLR}}-L_{\mathrm{AGN}}$ relation does not always follow a tight power law, and that more complex physical processes may contribute significantly to the scatter. In order to further investigate the single-object $R_{\mathrm{BLR}}-L_{\mathrm{AGN}}$ relation, repeated monitoring campaigns for individual AGNs are needed to track the behavior of each object over a range of timescales and luminosity states. This will, in turn, help improve our understanding of the global $R_{\mathrm{BLR}}-L_{\mathrm{AGN}}$ relation.

\section{Summary}

We present the results of an optical spectroscopic monitoring program in 2014 targeting the galaxy NGC 5548 as part of the AGN STORM project. Our campaign spanned 6 months and observed the AGN with an almost daily cadence. Our main findings are as follows.

(1) We determined $\mathrm{H} \beta$ and He II $\lambda 4686$ emission-line lags relative to the far-UV and optical continua and found that the lag measured against the UV continuum is $\sim 2$ days longer than that measured against the optical continuum, consistent with the lag between the UV and optical continua. Given that past measurements of the $\mathrm{H} \beta$ lag against the optical continuum for this object range from $\sim 4$ to $\sim 25$ days and assuming that this 2 day lag difference is constant over time, the characteristic size of the BLR inferred from previous data is biased low by $10 \%-50 \%$. Depending on how the ratio of UV and optical $\mathrm{H} \beta$ lags scales with other AGN properties, the RM BH mass scale and the $R_{\mathrm{BLR}}-L_{\mathrm{AGN}}$ relation may be affected, which would, in turn, impact single-epoch $M_{\mathrm{BH}}$ estimates for high-redshift AGNs.

(2) We measured velocity-resolved lags for the broad $\mathrm{H} \beta$ line and found a double-peaked lag profile as a function of line-of-sight velocity, with shorter lags in the highvelocity wings. The overall shape of the lag profile is qualitatively similar to those of Keplerian models (e.g., Horne et al. 2004) and is very similar to what is found for Ly $\alpha$ (De Rosa et al. 2015). 
(3) Both the $\mathrm{H} \beta$ and He II $\lambda 4686$ emission lines exhibit significant changes in their response to UV continuum variations halfway through our monitoring campaign. The line light curves decorrelate from that of the continuum and remain in a suppressed state until near the end of the campaign. The same anomalous behavior is observed for all the UV emission lines (Papers I and IV). Further investigation into the simultaneous UV and optical line responses during this campaign may elucidate the cause of this anomaly. Depending on how frequently this phenomenon occurs in the AGN population as a whole, this effect could contribute to the scatter in both single-object and global $R_{\mathrm{BLR}}-L_{\mathrm{AGN}}$ relations. This type of anomalous line behavior is likely only detectable with monitoring campaigns that have a combination of high cadence, long duration, and high data quality.

(4) Given the optical luminosity of NGC 5548 during our campaign, the $\mathrm{H} \beta$ lag measured against the optical continuum is a factor of five shorter than the expected value based on the $R_{\mathrm{BLR}}-L_{\mathrm{AGN}}$ relation for NGC 5548 from past monitoring campaigns. Our results, combined with other recent $\mathrm{H} \beta$ lag measurements, suggest that this object does not follow a simple power-law $R_{\mathrm{BLR}}-L_{\mathrm{AGN}}$ relation at all times.

We thank the staffs at the various observatories used to obtain the data in this paper. Support for HST program no. GO13330 was provided by NASA through a grant from the Space Telescope Science Institute, which is operated by the Association of Universities for Research in Astronomy, Inc., under NASA contract NAS5-26555. L.P. and A.J.B. have been supported by National Science Foundation (NSF) grant AST1412693. M.M.F., G.D.R., B.M.P., C.J.G., and R.W.P. are grateful for the support of NSF grant AST-1008882 to The Ohio State University. M.C. Bentz gratefully acknowledges support through NSF CAREER grant AST-1253702 to Georgia State University. A.V.F.'s group at UC Berkeley is grateful for financial assistance from NSF grant AST-1211916, the TABASGO Foundation, and the Christopher R Redlich Fund. C.S.K. is supported by NSF grant AST-1515876. V.N.B. gratefully acknowledges assistance from NSF Research at Undergraduate Institutions (RUI) grant AST-1312296. M.C. Bottorff acknowledges HHMI for support through an undergraduate science education grant to Southwestern University. K.D.D. is supported by an NSF Fellowship awarded under grant AST-1302093. M.E. thanks the members of the Center for Relativistic Astrophysics at Georgia Tech and the Department of Astronomy at the University of Washington, where he was based during the observing campaign, for their warm hospitality. R.E. gratefully acknowledges support from NASA under awards NNX13AC26G, NNX13AC63G, and NNX13AE99G. J.M.G. gratefully acknowledges support from NASA under award NNH13CH61C. P.B.H. is supported by NSERC. K.H. acknowledges support from the UK Science and Technology Facilities Council through grant ST/M001296/1. T.W.-S.H. is supported by the DOE Computational Science Graduate Fellowship, grant no. DE-FG02-97ER25308. M.I. acknowledges support from the Creative Initiative program, no. 2008-0060544, of the National Research Foundation of Korea (NRFK) funded by the Korean government (MSIP). M.D.J. acknowledges NSF grant AST-0618209. SRON is financially supported by NWO, the Netherlands Organization for
Scientific Research. B.C.K. is partially supported by the UC Center for Galaxy Evolution. C.S.K. acknowledges the support of NSF grant AST-1009756. D.C.L. acknowledges support from NSF grants AST-1009571 and AST-1210311. P.L. acknowledges support from Fondecyt grant no. 1161184. A. P. acknowledges support from an NSF graduate fellowship and a UCSB Deans Fellowship. J.S.S. acknowledges CNPq, National Council for Scientific and Technological Development (Brazil) for partial support and The Ohio State University for warm hospitality. N.T. acknowledges support from CONICYT PAI/82140055. T.T. has been supported by NSF grant AST-1412315. T.T. and B.C.K. acknowledge support from the Packard Foundation in the form of a Packard Research Fellowship to T.T. Also, T.T. thanks the American Academy in Rome and the Observatory of Monteporzio Catone for kind hospitality. The Dark Cosmology Centre is funded by the Danish National Research Foundation. M.V. gratefully acknowledges support from the Danish Council for Independent Research via grant no. DFF 4002-00275. J.-H.W. acknowledges support by the National Research Foundation of Korea (NRF) grant funded by the Korean government (no. 2010-0027910). This work is based partly on observations obtained with the Apache Point Observatory $3.5 \mathrm{~m}$ telescope, which is owned and operated by the Astrophysical Research Consortium. Research at Lick Observatory is partially supported by a generous gift from Google. This research has made use of the NASA/IPAC Extragalactic Database (NED), which is operated by the Jet Propulsion Laboratory, California Institute of Technology, under contract with the National Aeronautics and Space Administration. We thank the anonymous referee for the thorough review and comments that helped improve the clarity of this manuscript.

\section{References}

Antonucci, R. R. J., \& Cohen, R. D. 1983, ApJ, 271, 564

Baldwin, J. A. 1977, ApJ, 214, 679

Barth, A. J., Bennert, V. N., Canalizo, G., et al. 2015, ApJS, 217, 26

Barth, A. J., \& Bentz, M. C. 2016, MNRAS, 458, L109

Barth, A. J., Nguyen, M. L., Malkan, M. A., et al. 2011, ApJ, 732, 121

Barth, A. J., Pancoast, A., Bennert, V. N., et al. 2013, ApJ, 769, 128

Bentz, M. C., Denney, K. D., Cackett, E. M., et al. 2007, ApJ, 662, 205

Bentz, M. C., Denney, K. D., Grier, C. J., et al. 2013, ApJ, 767, 149

Bentz, M. C., Horne, K., Barth, A. J., et al. 2010a, ApJL, 720, L46

Bentz, M. C., \& Katz, S. 2015, PASP, 127, 67

Bentz, M. C., Peterson, B. M., Netzer, H., Pogge, R. W., \& Vestergaard, M. 2009a, ApJ, 697, 160

Bentz, M. C., Peterson, B. M., Pogge, R. W., Vestergaard, M., \& Onken, C. A. 2006, ApJ, 644, 133

Bentz, M. C., Walsh, J. L., Barth, A. J., et al. 2009b, ApJ, 705, 199

Bentz, M. C., Walsh, J. L., Barth, A. J., et al. 2010b, ApJ, 716, 993

Blandford, R. D., \& McKee, C. F. 1982, ApJ, 255, 419

Boroson, T. A., \& Green, R. F. 1992, ApJS, 80, 109

Bruzual, G., \& Charlot, S. 2003, MNRAS, 344, 1000

Clavel, J., Reichert, G. A., Alloin, D., et al. 1991, ApJ, 366, 64

Collier, S., \& Peterson, B. M. 2001, ApJ, 555, 775

Collier, S. J., Horne, K., Kaspi, S., et al. 1998, ApJ, 500, 162

Collin, S., Kawaguchi, T., Peterson, B. M., \& Vestergaard, M. 2006, A\&A, 456, 75

Crenshaw, D. M., \& Blackwell, J. H., Jr. 1990, ApJL, 358, L37

Czerny, B., Doroshenko, V. T., Nikołajuk, M., et al. 2003, MNRAS, 342, 1222

Czerny, B., Schwarzenberg-Czerny, A., \& Loska, Z. 1999, MNRAS, 303, 148 De Rosa, G., Peterson, B. M., Ely, J., et al. 2015, ApJ, 806, 128

Denney, K. D., Bentz, M. C., Peterson, B. M., et al. 2006, ApJ, 653, 152 Denney, K. D., Peterson, B. M., Pogge, R. W., et al. 2009, ApJL, 704, L80

Denney, K. D., Peterson, B. M., Pogge, R. W., et al. 2010, ApJ, 721, 715

Dietrich, M., Kollatschny, W., Peterson, B. M., et al. 1993, ApJ, 408, 416

Dietrich, M., Peterson, B. M., Albrecht, P., et al. 1998, ApJS, 115, 185

Done, C., \& Krolik, J. H. 1996, ApJ, 463, 144 
Du, P., Hu, C., Lu, K.-X., et al. 2015, ApJ, 806, 22

Du, P., Lu, K.-X., Hu, C., et al. 2016a, arXiv:1602.01922

Du, P., Lu, K.-X., Zhang, Z.-X., et al. 2016b, arXiv:1604.06218

Edelson, R., Gelbord, J. M., Horne, K., et al. 2015, ApJ, 806, 129

Emmering, R. T., Blandford, R. D., \& Shlosman, I. 1992, ApJ, 385, 460

Fausnaugh, M. M. 2016, arXiv:1609.04014

Fausnaugh, M. M., Denney, K. D., Barth, A. J., et al. 2016, ApJ, 821, 56

Filippenko, A. V. 1982, PASP, 94, 715

Gaskell, C. M. 1988, ApJ, 325, 114

Gaskell, C. M., \& Goosmann, R. W. 2013, ApJ, 769, 30

Gaskell, C. M., \& Goosmann, R. W. 2016, Ap\&SS, 361, 67

Gaskell, C. M., \& Sparke, L. S. 1986, ApJ, 305, 175

Gilbert, K. M., \& Peterson, B. M. 2003, ApJ, 587, 123

Goad, M., \& Wanders, I. 1996, ApJ, 469, 113

Goad, M. R., \& Korista, K. T. 2014, MNRAS, 444, 43

Goad, M. R., Korista, K. T., De Rosa, G., et al. 2016, ApJ, 824, 11

Goad, M. R., Korista, K. T., \& Knigge, C. 2004, MNRAS, 352, 277

Goad, M. R., Korista, K. T., \& Ruff, A. J. 2012, MNRAS, 426, 3086

Graham, A. W., Onken, C. A., Athanassoula, E., \& Combes, F. 2011, MNRAS, 412, 2211

Grier, C. J., Martini, P., Watson, L. C., et al. 2013, ApJ, 773, 90

Ho, L. C., \& Kim, M. 2015, ApJ, 809, 123

Horne, K. 1986, PASP, 98, 609

Horne, K., Peterson, B. M., Collier, S. J., \& Netzer, H. 2004, PASP, 116, 465

Horne, K., Welsh, W. F., \& Peterson, B. M. 1991, ApJL, 367, L5

Kaspi, S., Brandt, W. N., Maoz, D., et al. 2007, ApJ, 659, 997

Kaspi, S., Maoz, D., Netzer, H., et al. 2005, ApJ, 629, 61

Kaspi, S., Smith, P. S., Netzer, H., et al. 2000, ApJ, 533, 631

Kelly, B. C., Bechtold, J., \& Siemiginowska, A. 2009, ApJ, 698, 895

Kilerci Eser, E., Vestergaard, M., Peterson, B. M., Denney, K. D., \& Bentz, M. C. 2015, ApJ, 801, 8

King, A. L., Martini, P., Davis, T. M., et al. 2015, MNRAS, 453, 1701

Kollatschny, W. 2003, A\&A, 407, 461

Korista, K. T., Alloin, D., Barr, P., et al. 1995, ApJS, 97, 285

Kormendy, J., \& Ho, L. C. 2013, ARA\&A, 51, 511

Kovačević, J., Popović, L. Č., \& Dimitrijević, M. S. 2010, ApJS, 189, 15

Kozłowski, S., Kochanek, C. S., Udalski, A., et al. 2010, ApJ, 708, 927

Krolik, J. H., Horne, K., Kallman, T. R., et al. 1991, ApJ, 371, 541

Laor, A. 1998, ApJL, 505, L83

Leighly, K. M., \& Moore, J. R. 2004, ApJ, 611, 107

Li, Y.-R., Wang, J.-M., Ho, L. C., Du, P., \& Bai, J.-M. 2013, ApJ, 779, 110

Lu, K.-X., Du, P., Hu, C., et al. 2016, ApJ, 827, 118

MacLeod, C. L., Ivezić, Ž., Kochanek, C. S., et al. 2010, ApJ, 721, 1014

McHardy, I. M., Cameron, D. T., Dwelly, T., et al. 2014, MNRAS, 444, 1469

McLure, R. J., \& Jarvis, M. J. 2002, MNRAS, 337, 109

Mejía-Restrepo, J. E., Trakhtenbrot, B., Lira, P., Netzer, H., \& Capellupo, D. M. 2016, MNRAS, 460, 187

Miller, J. S., \& Stone, R. P. S. 1993, Lick Observatory, Tech. Rep. (Santa Cruz, CA: Lick Observatory)

Murray, N., \& Chiang, J. 1997, ApJ, 474, 91

Onken, C. A., Ferrarese, L., Merritt, D., et al. 2004, ApJ, 615, 645

Pancoast, A., Brewer, B. J., \& Treu, T. 2011, ApJ, 730, 139

Pancoast, A., Brewer, B. J., Treu, T., et al. 2014, MNRAS, 445, 3073
Park, D., Kelly, B. C., Woo, J.-H., \& Treu, T. 2012a, ApJS, 203, 6

Park, D., Woo, J.-H., Treu, T., et al. 2012b, ApJ, 747, 30

Peterson, B. M. 1993, PASP, 105, 247

Peterson, B. M. 2006, in ASP Conf. Ser. 360 AGN Variability from X-Rays to Radio Waves, ed. C. M. Gaskell et al. (San Francisco, CA: ASP), 191

Peterson, B. M. 2014, SSRv, 183, 253

Peterson, B. M., Balonek, T. J., Barker, E. S., et al. 1991, ApJ, 368, 119

Peterson, B. M., Barth, A. J., Berlind, P., et al. 1999, ApJ, 510, 659

Peterson, B. M., Berlind, P., Bertram, R., et al. 2002, ApJ, 581, 197

Peterson, B. M., Denney, K. D., De Rosa, G., et al. 2013, ApJ, 779, 109

Peterson, B. M., Ferrarese, L., Gilbert, K. M., et al. 2004, ApJ, 613, 682

Peterson, B. M., \& Horne, K. 2004, AN, 325, 248

Peterson, B. M., Pogge, R. W., Wanders, I., Smith, S. M., \& Romanishin, W. 1995, PASP, 107, 579

Peterson, B. M., Wanders, I., Bertram, R., et al. 1998, ApJ, 501, 82

Pogge, R. W., \& Peterson, B. M. 1992, AJ, 103, 1084

Reichert, G. A., Rodriguez-Pascual, P. M., Alloin, D., et al. 1994, ApJ, 425, 582

Rosenblatt, E. I., \& Malkan, M. A. 1990, ApJ, 350, 132

Santos-Lleó, M., Chatzichristou, E., de Oliveira, C. M., et al. 1997, ApJS, 112,271

Schlafly, E. F., \& Finkbeiner, D. P. 2011, ApJ, 737, 103

Schlegel, D. J., Finkbeiner, D. P., \& Davis, M. 1998, ApJ, 500, 525

Sergeev, S. G., Doroshenko, V. T., Golubinskiy, Y. V., Merkulova, N. I., \& Sergeeva, E. A. 2005, ApJ, 622, 129

Shappee, B. J., Prieto, J. L., Grupe, D., et al. 2014, ApJ, 788, 48

Shen, Y., Horne, K., Grier, C. J., et al. 2016, ApJ, 818, 30

Skielboe, A., Pancoast, A., Treu, T., et al. 2015, MNRAS, 454, 144

Stirpe, G. M., Winge, C., Altieri, B., et al. 1994, ApJ, 425, 609

Ulrich, M. H., Boksenberg, A., Bromage, G. E., et al. 1984, MNRAS, 206, 221

Ulrich, M.-H., \& Horne, K. 1996, MNRAS, 283, 748

van der Marel, R. P., \& Franx, M. 1993, ApJ, 407, 525

van Dokkum, P. G. 2001, PASP, 113, 1420

van Groningen, E., \& Wanders, I. 1992, PASP, 104, 700

Vasudevan, R. V., Fabian, A. C., Gandhi, P., Winter, L. M., \& Mushotzky, R. F. 2010, MNRAS, 402, 1081

Véron-Cetty, M.-P., Joly, M., \& Véron, P. 2004, A\&A, 417, 515

Vestergaard, M., \& Peterson, B. M. 2006, ApJ, 641, 689

Wandel, A., Peterson, B. M., \& Malkan, M. A. 1999, ApJ, 526, 579

Wanders, I., Goad, M. R., Korista, K. T., et al. 1995, ApJL, 453, L87

Wanders, I., Peterson, B. M., Alloin, D., et al. 1997, ApJS, 113, 69

Watson, L. C., Mathur, S., \& Grupe, D. 2007, AJ, 133, 2435

Welsh, W. F. 1999, PASP, 111, 1347

Welsh, W. F., \& Horne, K. 1991, ApJ, 379, 586

Welsh, W. F., Martino, D. L., Kawaguchi, G., \& Kollatschny, W. 2007, in ASP

Conf. Ser. 373, The Central Engine of Active Galactic Nuclei, ed. L. C. Ho \& J.-W. Wang (San Francisco, CA: ASP), 29

White, R. J., \& Peterson, B. M. 1994, PASP, 106, 879

Whittle, M. 1992, ApJS, 79, 49

Woo, J.-H., Treu, T., Barth, A. J., et al. 2010, ApJ, 716, 269

Woo, J.-H., Yoon, Y., Park, S., Park, D., \& Kim, S. C. 2015, ApJ, 801, 38

Zu, Y., Kochanek, C. S., Kozłowski, S., \& Udalski, A. 2013, ApJ, 765, 106

Zu, Y., Kochanek, C. S., \& Peterson, B. M. 2011, ApJ, 735, 80 